\title{
High-Fidelity CFD Simulation of Wave Run-Up for Single/Multiple Surface-Piercing Cylinders in Regular Head Waves
}

\author{
Sung-Hwan Yoon ${ }^{\dagger}$, Dong-Hwan Kim ${ }^{\dagger}$, Hamid Sadat-Hosseini, \\ Jianming Yang, Frederick Stern* \\ IIHR-Hydroscience \& Engineering, University of Iowa, Iowa City, IA 52242-1585, USA
}

\begin{abstract}
The capabilities of CFDShip-Iowa V4.5 and V6.2 simulations for wave run-up for single/multiple surface-piercing cylinders in regular waves are assessed in conjunction with the ITTC OEC Workshop on VIV and Wave Run-up held in Nantes, France October 17-18, 2013. Statistical convergence and grid/domain/turbulence model sensitivity studies show uncertainty $U_{I}$ values are less than $1 \%$ for both the experiment/simulation data and grid/domain/turbulence model sensitivities are less than the facility bias $\mathrm{U}_{\mathrm{FB}}$. Simulations are qualitatively validated using $\mathrm{U}_{\mathrm{FB}}$ and error $\mathrm{E}$ between experiment and simulation. Errors for single/four cylinder(s) are comparable with $\mathrm{U}_{\mathrm{FB}}$ and average error $\overline{\mathrm{E}}$ for the other CFD data of the workshop participants. Results from both V4.5 and V6.2 agree well with experiment and also show capabilities to apply for complex/large-scale problem and to describe detailed wave breaking/spraying for V4.5 and V6.2, respectively. Study on run-up profile shows mean wave elevations are similar to free surface elevations for a cylinder in a steady current. For wave steepness/wave length effect, $2^{\text {nd }}$ to $1^{\text {st }}$ harmonic ratio on the cylinder weather face decreases with steepness for short wave, but increases for long wave. Wave diffraction analysis shows that $2^{\text {nd }} / 3^{\text {rd }}$ to $1^{\text {st }}$ harmonic ratio in diffracted wave contour reach up to $18 \sim 41 \%$ / 9 16\% respectively. Due to interaction between cylinders, crest/trough heights
\end{abstract}

Co-first authors
* Corresponding author; Tel.: +1 319335 5215; fax: +1 319335 5238; e-mail address: frederick-stern@ uiowa.edu 
increase $5-17 \%$ / 4-10\% for 4C, respectively. Study on vortex shedding shows short wave has insufficient time to develop periodic vortex shedding compared to long wave.

Key words: CFD, run-up, wave diffraction, vortex shedding

\section{Introduction}

Accurate estimation of run-up height and forces induced by ocean waves is essential to the design of offshore platforms. Attention has focused on interactions between steep waves and floating platform systems, for which a typical geometry consists of single/multiple surface-piercing cylinders.

Potential flow methods are the common numerical approach to study the run-up height and forces. Since McCamy and Fuchs [1] presented an analytic solution for a bottom-mounted circular cylinder, further advances based on linear potential theory have been made [2]. For steep waves, the linear assumption significantly under-predicts crest heights around columns [3]. Second-order and higher-order effects have been studied [4], but non-linear tools based on potential theory have limitations due to inability to model wave breaking and viscous effects in simulating strongly non-linear wave-body interaction flows [5].

CFD simulations which include viscous effects have recently been used to study the non-linear mechanism of wave run-up [6] [7], which showed that fully nonlinear CFD simulation can capture highly nonlinear wave-structure interaction effects, such as jet-like run-up that may occur on the weather side of platform columns, which can not be predicted by potential. In order to assess the capabilities of state-ofthe-art CFD codes for wave run-up simulation around cylinders, the ITTC Ocean Engineering Committee (OEC) held Workshop on VIV and Wave Run-up in Nantes, France October 17-18, 2013 for blind submissions for benchmark tests [8]. Numerical results were compared with experimental data measured from MOERI [9] and MARINTEK [10] for single (1C) and four circular cylinders (4C), respectively. For $1 \mathrm{C}$ the CFD results at the weather side of cylinder agreed well with the experimental results for the $1^{\text {st }}$

harmonics of wave elevation, but not for mean and the $2^{\text {nd }}$ harmonics. Overall trends of experimental 
results by MOERI and MARINTEK [4] for $1 \mathrm{C}$ were also similar for the $1^{\text {st }}$ harmonic of wave elevation, but mean and the $2^{\text {nd }}$ harmonics were different in values and in trend.

Herein, the capabilities of CFDShip-Iowa V4.5 [11] and V6.2 [12] simulations for wave run-up for single/multiple surface-piercing cylinders in regular waves are assessed. Pre (blind) and post workshop results were submitted to the OEC. The simulations are for full-scale conditions, as specified by the OEC. Quantitative verification and validation [13] studies are beyond the scope since the grid sizes are large and experimental uncertainties are not available. However, statistical convergence and grid, domain and turbulence model sensitivity are evaluated and the simulations are qualitatively validated using comparison error $\mathrm{E}=(\mathrm{D}-\mathrm{S}) \% \mathrm{D}$ where $\mathrm{D}$ and $\mathrm{S}$ are the experimental and simulation values, respectively. Wave elevations and horizontal/vertical forces experimental data was provided post workshop by the OEC. The facility biases $U_{\mathrm{FB}}$ for wave elevations based on the differences between the MOERI [9] and MARINTEK [4] data for $1 \mathrm{C}$ short and long waves for $0^{\text {th }}, 1^{\text {st }}$ and $2^{\text {nd }}$ harmonics are compared with the sensitivity studies and validation results. Variables are grouped into first ( $1^{\text {st }}$ harmonics $)$ and second $\left(0^{\text {th }}\right.$ and $2^{\text {nd }}$ harmonics) order terms for separate assessment capabilities both for the experiments and CFD. The effects of wave steepness and length, wave diffraction, interaction between cylinders, and vortex shedding are studied.

\section{Experimental Validation Data}

The ITTC OEC Workshop used experimental data from MOERI [9] wave basin $(56 \times 30 \times 4.5 \mathrm{~m})$ for six wavelengths $(\lambda / d=4.7,6.2,7.9,9.7,14.0,21.9)$ and four wave steepness $(H / \lambda=1 / 50,1 / 30,1 / 16,1 / 10)$ conditions where $\lambda$ and $H$ are the wave length and height, respectively, and $d$ is the cylinder diameter. Table 1 provides the $1 \mathrm{C}$ model and full-scale geometries. The model scale (1/50) and aspect ratio (Draft/Diameter=1.5) are small. The OEC provided time histories of wave elevations $\eta(\mathrm{t})$ and horizontal $X(\mathrm{t})$ and vertical $\mathrm{Z}(\mathrm{t})$ forces and their $0^{\text {th }}, 1^{\text {st }}$ and $2^{\text {nd }}$ harmonics for selected test cases. Figure 1(a) illustrates the locations of the wave gages near the cylinder. Ten gages are located at $r=0.513 d$ and $r=1.0 d$ 
at angles $\theta=0^{\circ}, 45^{\circ}, 90^{\circ}, 135^{\circ}, 180^{\circ}$. Regular/deep-water (water depth/wavelength $>1 / 2$ ) waves with zero heading $\beta=0$ are generated as the incident waves for all tests. Table 1 includes the $\mathrm{U}_{\mathrm{FB}}=\left(\mathrm{D}_{1}-\mathrm{D}_{2}\right) \% \mathrm{D}_{1}$ where $\mathrm{D}_{1}$ and $\mathrm{D}_{2}$ are the MOERI [9] and MARINTEK [4] wave elevation data, which is $4.7 \%$ and $118 \%$ for the $1^{\text {st }}$ and $2^{\text {nd }}$ order variables, respectively.

The ITTC OEC Workshop used experimental data from MARINTEK [10] Towing tank III $(80 \times 10.5 \times 10 \mathrm{~m})$ for four wavelengths $(\lambda / d=4.7,7.9,14.0,21.9)$ and three wave steepness $(H / \lambda=1 / 30$, $1 / 16,1 / 10$ ) conditions for the $4 \mathrm{C}$ validation. Table 1 provides the $4 \mathrm{C}$ model and full-scale geometries. The model scale (1/49) and aspect ratio (1.5) are small. The OEC provided time histories of wave elevations $\eta(\mathrm{t})$ and their $0^{\text {th }}, 1^{\text {st }}$ and $2^{\text {nd }}$ harmonics for selected test cases. The arrangement of the four cylinders and the locations of wave probes are shown in Figure 1(b) and 1(c). Wave elevations around down wave cylinder (denoted as $A$ ) were measured using twelve gages. Four gages are located along each radial line at angles $180^{\circ}, 225^{\circ}$, and $270^{\circ}$ relative to the incoming waves. The incoming waves are regular waves with $\beta=0^{\circ}$ and $\beta=45^{\circ}$ heading and with deep-water condition.

\section{Computational Methods, Conditions and Grids}

The CFDShip-Iowa V4.5 is an overset, block structured CFD solver using either absolute or relative inertial non-orthogonal curvilinear coordinate system for arbitrary moving but non-deforming control

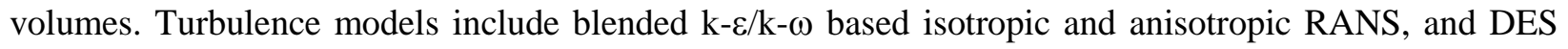
approaches with near-wall or wall functions. A single-phase level-set method is used for free-surface capturing with air/water interface modeled using a semi-coupled method. Captive, semi-captive, and full 6DOF capabilities for multi-objects with parent/child hierarchy are available.

Table 2 summarizes the V4.5 simulations. For $1 \mathrm{C}$, the shortest $\lambda / d=4.7$ and the longest $\lambda / d=21.9$ waves with $H / \lambda=1 / 30,1 / 16$ and $1 / 10$ were used. For $4 \mathrm{C}$, the shortest $\lambda / d=4.7$ wave with $H / \lambda=1 / 30$ and $\beta=0^{\circ}$ was used. Table 2 includes the full-scale Reynolds $\operatorname{Re}=\bar{u} d / v$ and Froude $\mathrm{Fr}=\bar{u} / \sqrt{g d}$ numbers based on the wave-induced mean velocity (defined later) and cylinder diameter. In context of ship 
hydrodynamics: the Re is model scale; the Fr is very small; and the wavelength and steepness are large. In context of single-phase cylinder flow all Re are in the post-critical regime.

The results submitted to the ITTC OEC Workshop used grids G1, G2 and G8 for the 1C short and long $\lambda / d$ and $4 \mathrm{C}$ short $\lambda / d$ test cases, respectively. G3 (fine) and G4 (medium) with same domain size (medium) but different grid resolution were used to study grid sensitivity: $\Delta \mathrm{G} \% \mathrm{G} 3$. G4 has similar grid resolution as G1 and G2. Domain size study used G1 (D1), G4 (D2) and G5 (D3), which have large, medium and small upstream lengths, respectively, but the same domain sizes in the other directions: $\Delta \mathrm{D} \% \mathrm{D} 1 . \lambda / \Delta x$ and $H / \Delta z$ are more than 75 and 20 respectively for all cases and the same resolutions are maintained for domain size study to eliminate grid resolution effects. G6 used for comparison of URANS and DES turbulence models: $\triangle \mathrm{URANS} \% \mathrm{DES}$. The total number of grid points of G6 is almost the same with G1 and G2, but it has much smaller domain size and fine enough grid resolution for the DES model.

Figure 2 shows overset grid system and boundary condition for V4.5 simulation. The cylinder surface is a no-slip wall. Zero gradient boundary conditions are applied on the top, bottom and both sides of the domain. Overset grids are used for grid clustering. The grid system consists of a 'boundary layer grid', ' $1{ }^{\text {st }}$ refinement grid', ' $2^{\text {nd }}$ refinement grid' and a 'background grid'. From the 'boundary layer grid' to the 'background grid', grid sizes increase gradually, ensuring tolerable numerical errors in overset interpolations. The $1 \mathrm{C}$ and $4 \mathrm{C}$ grid sizes are large ranging from $13-26 \mathrm{M}$ and $65 \mathrm{M}$, respectively.

At the inlet and outlet, $2^{\text {nd }}$ order Stoke's waves are imposed for non-dimensional free surface elevation, velocities and pressure. For the deep-water condition, the equations are:

$$
\begin{aligned}
& \eta_{\mathrm{I}}(x, t)=A_{1} \cos \theta+\frac{A_{1}^{2} k}{2} \cos 2 \theta \\
& u_{\mathrm{I}}(x, z, t)=\frac{A_{1} k}{F r \sqrt{k}} e^{k z} \cos \theta+\frac{6 A_{1}^{2} k^{2}}{F r \sqrt{k}} e^{2 k\left(z-d_{w}\right)} \cos 2 \theta \\
& w_{\mathrm{I}}(x, z, t)=\frac{A_{1} k}{F r \sqrt{k}} e^{k z} \sin \theta+\frac{3}{4} \frac{A_{1}^{2} k^{2}}{F r \sqrt{k}} e^{2 k\left(z-d_{w}\right)} \sin 2 \theta \\
& p_{\mathrm{I}}(x, z, t)=\frac{A_{1}}{F r^{2}} e^{k z} \cos \theta+\frac{6 A_{1}^{2} k}{F r^{2}} e^{2 k\left(z-d_{w}\right)} \cos 2 \theta-\frac{1}{2} \frac{A_{1}^{2} k}{F r^{2}} e^{2 k z}
\end{aligned}
$$


where $\theta=k x-\omega t . A_{1}, k, \omega$ and $d$ are the linear part of incident wave amplitude, wave number, wave angular frequency and water depth, respectively.

CFDShip-Iowa V6.2 utilizes the filtered incompressible Navier-Stokes equations fully transformed from Cartesian coordinates to orthogonal curvilinear coordinates. Extended to multi-block topology, V6.2 has been improved for its capability to handle realistic geometries remarkably. V6.2 is equipped with a dynamic SGS turbulence model for LES computation. In present study, however, no SGS model was used for comparison with URANS/DES model in V4.5. The air/water interface is calculated by VOF method with a level-set based sharp interface treatment.

As shown in Table 2, V6.2 simulations were conducted using G7 for the short wave $\lambda / d=4.7$ with $H / \lambda=1 / 16$ and $1 / 10$ conditions. Since V6.2 is based on an orthogonal curvilinear grid solver, cylindrical grid system is required as shown in Fig. 3. G7 has a cylindrical topology with radius of $2 \lambda$. The far boundary in the radial direction $\left(i_{\max }\right.$ plane) is split into two parts, inlet and outlet, and then the incoming wave and convective boundary condition are applied for inlet and outlet, respectively. Bottom/top sides are slip walls. Periodic boundary condition is imposed at a plane shared by $j_{\min }$ and $j_{\max }$ planes in azimuthal direction. The $1 \mathrm{C}$ grid size is $11 \mathrm{M}$, which is $54 \%$ smaller than G1. Due to different solver characteristics for V4.5 and V6.2, computational domain and boundary condition are not same with those of V4.5. However, preliminary tests without cylinder have shown the validity of present domain size/grid resolution/boundary condition for each V4.5 and V6.2 solver in steep wave conditions, although not presented herein.

In order to describe highly steep incident wave more accurately, numerical tests for the incident wave boundary condition in V6.2 were performed. Without cylinder in a domain $(\mathrm{L} \times \mathrm{H} \times \mathrm{W}=3 \lambda \times 2 \lambda \times \lambda), 2^{\text {nd }}$ and $5^{\text {th }}$ order Stoke's wave were imposed as incident wave boundary condition and the CFD wave elevation at the center of the domain were compared with the elevations from the corresponding incident wave formula. For $H / \lambda=1 / 16$ and $1 / 10$ cases, $2^{\text {nd }}$ and $5^{\text {th }}$ order Stoke's wave formula showed differences in crest height and phase. In addition, for $H / \lambda=1 / 10$ case, the differences in crest height / phase between the CFD 
data and the corresponding incident wave formula were $8 \% / 18 \% 360^{\circ}$ and $3.8 \% / 1.9 \% 360^{\circ}$ for $2^{\text {nd }}$ and $5^{\text {th }}$ order Stoke's wave, respectively. Thus, in order to minimize difference between incident wave and actual CFD computation, $5^{\text {th }}$ order Stoke's wave is used in V6.2 for the inlet elevation and velocities. Whereas V4.5 requires pressure boundary condition for incident wave, V6.2 utilizes homogeneous Neumann boundary condition without explicit description of pressure. For deep-water condition, free surface elevation and velocities of incident wave are given by:

$$
\begin{aligned}
& \eta_{\mathrm{I}}(x, t)=\frac{1}{k} \sum_{n=1}^{5} \epsilon^{n} b_{n} \cos (n \theta) \\
& u_{\mathrm{I}}(x, z, t)=C_{0} \sqrt{\frac{g}{k}} \sum_{n=1}^{5} \epsilon^{n} n a_{n} \cosh (n k z) \cos (n \theta) \\
& w_{\mathrm{I}}(x, z, t)=C_{0} \sqrt{\frac{g}{k}} \sum_{n=1}^{5} \epsilon^{n} n a_{n} \sinh (n k z) \sin (n \theta)
\end{aligned}
$$

where $\epsilon=k A_{1}$ and $\theta=k x-\omega t . A_{1}, k$ and $\omega$ are the linear part of incident wave amplitude, wave number and wave angular frequency, respectively. Coefficients $a_{n}$ and $b_{n}$ are given in [14].

\section{Statistical Convergence, Harmonic Analysis and Grid, Domain and Turbulence Model Sensitivity}

Simulations were conducted for 10 wave periods, as specified by the OEC. Figure 4 and 5 compare experiments and simulation time histories for wave elevation and horizontal/vertical forces for $1 \mathrm{C}$ and $\lambda / d=4.7, H / \lambda=1 / 30$ and $\lambda / d=21.9, H / \lambda=1 / 10$, respectively, including running mean and running RMS, which are typical for all the results. Statistical convergence uncertainty is based on the magnitude of the

running mean and running RMS oscillations $U_{I}=\left|\frac{1}{2}\left(S_{U}-S_{L}\right)\right|$, where $S_{U}$ and $S_{L}$ are the maximum and minimum value, respectively [13]. The running RMS $\mathrm{U}_{\mathrm{I}}$ for the experiment / CFD are $0.48 \% A_{1}, 0.29 \% X_{1}$, $0.54 \% Z_{1} / 0.54 \% A_{1}, 0.28 \% X_{1} 0.66 \% Z_{1}$ for $\lambda / d=4.7$ and $0.58 \% A_{1}, 0.75 \% X_{1}, 0.74 \% Z_{1} / 0.24 \% A_{1}, 0.23 \% X_{1}$, $0.17 \% Z_{1}$ for $\lambda / d=21.9$. The $\mathrm{U}_{\mathrm{I}}$ values are less than $1 \%$ for both the experiments and simulations.

Fast Fourier transform (FFT) was conducted to have harmonics of wave elevation $\left(\eta_{0}, \eta_{1}, \eta_{2}\right)$ and horizontal $\left(X_{0}, X_{1}, X_{2}\right) /$ vertical forces $\left(Z_{0}, Z_{1}, Z_{2}\right)$. For harmonic analysis, in order to exclude transition 
period, the last 7 and 5 wave periods out of the 10 cycles were chosen for short and long wave cases, respectively.

As shown in Table 3 , the grid sensitivity $\Delta \mathrm{G} \% \mathrm{G} 3$ for the $1^{\text {st }}$ and $2^{\text {nd }}$ order variables is $2 \%$ and $45 \%$, respectively. The domain size sensitivity $\Delta \mathrm{D} \% \mathrm{D} 1$ for $1^{\text {st }}$ and $2^{\text {nd }}$ order variables for $\mathrm{D} 2$ is $1 \%$ and $4 \%$, respectively, and for D3 is $7 \%$ and $22 \%$, respectively. The turbulence model sensitivity $\triangle$ URANS\%DES for $1^{\text {st }}$ and $2^{\text {nd }}$ order variables is $1 \%$ and $8 \%$, respectively. The grid, domain and turbulence model sensitivities are less than the respective $\mathrm{U}_{\mathrm{FB}}$ for first and second order variables, as shown in Table 1. Nonetheless, the $2^{\text {nd }}$ order variable sensitivity is large indicting the need for finer grids to resolve $2^{\text {nd }}$ order terms. The results indicate that both the large and medium domains should be sufficient for the validation studies, as used by G1, G2, G7 and G8. The turbulence model sensitivities are small such that the URANS turbulence model is sufficient for the validation studies.

\section{Validation}

For $1 \mathrm{C}$, the OEC provided validation data for the wave elevation and horizontal/vertical force time histories for short wave with $H / \lambda=1 / 30$ and long wave with $H / \lambda=1 / 10$, respectively, and their harmonics for short and long waves with all three $H / \lambda$. Short wave elevation time histories for $H / \lambda=1 / 16$ were extracted from [15]. For 4C, the OEC provided validation data for the wave elevation time histories and harmonics for the short wave with $H / \lambda=1 / 30$. In order to compare total elevations of the experiment and CFD, wave elevations at the crest $\bar{\eta}_{c}$ and trough $\bar{\eta}_{t}$ are calculated by averaging the maximum and minimum run-up heights for the last 10 cycles, respectively.

\subsection{Single circular cylinder (1C)}

Figure 6 shows that the $1^{\text {st }}$ harmonics of horizontal force decreases for increasing steepness, while the

$2^{\text {nd }}$ harmonics increases slightly for the short wave. Therefore, the $2^{\text {nd }}$ to $1^{\text {st }}$ harmonics ratio increases by increasing wave steepness. Long wave has lower $1^{\text {st }}$ harmonics, but higher $2^{\text {nd }}$ harmonics compared to 
short waves. Thus, nonlinearity in horizontal force is dominant for long wave cases. For vertical force, the $1^{\text {st }}$ and $2^{\text {nd }}$ harmonics are fairly independent of steepness. Table 4 shows that the E\%D values are 72 and $23 / 2 \% \mathrm{D}$ for $2^{\text {nd }}$ order and $1^{\text {st }}$ order amp/phase variables, respectively. The errors are mostly larger for the vertical than the horizontal force. The $\mathrm{X} / \mathrm{Z}$ phases lead the wave phase by small and large amounts for the short and long wave conditions. For the long wave condition, the $\mathrm{X} / \mathrm{Z}$ phases are nearly $180^{\circ}$ out of phase with the wave phase. Unfortunately, $\mathrm{U}_{\mathrm{FB}}$ for forces is not available for comparison.

Figure 7(a) shows the mean wave profile along the center plane from upstream, around cylinder, to downstream for $\lambda / d=4.7$ and $H / \lambda=1 / 30,1 / 16$ and $1 / 10$ conditions. The mean wave profiles of both experiment and CFD have higher run-up height on the front surface of cylinder than depression depth on the rear surface. Both results from V4.5 and V6.2 are reasonably accurate, even though discrepancies are observed on the weather and leeward faces. Along the cylinder shoulder, V6.2 predicts mean wave elevation for $H / \lambda=1 / 10$ case more accurately than V4.5. For V4.5, the elevations are below the calm water condition at upstream, but slightly higher than the calm water level at downstream locations. On the weather face of the cylinder, run-up heights for $H / \lambda=1 / 30$ and $1 / 16$ are similar, but increases for $H / \lambda=1 / 10$. Along the cylinder shoulder, depression pattern of V4.5 is similar for all three steepness. On the leeward face of the cylinder, the mean profiles of V4.5 are similar, but the elevation of V6.2 for $H / \lambda=1 / 10$ increases and oscillates at downstream likely due to wave breaking and no turbulence model.

Figure 7(b) shows the mean wave elevations along the center plane from upstream, around cylinder, to downstream for $\lambda / d=21.9$ and $H / \lambda=1 / 30,1 / 16$ and $1 / 10$ conditions. Compared to the experimental data, results from V4.5 are reasonably accurate except on the weather face for $H / \lambda=1 / 16$ and $1 / 10$ cases. For V4.5, the elevations are slightly higher than the calm water level at upstream and downstream locations. On the weather face of the cylinder, the run-up heights increase with increasing steepness and the height for $H / \lambda=1 / 10$ case is much higher than that for short wave. Along the shoulder and on the leeward face of the cylinder, the mean profiles are also similar for all three steepness. 
The mean wave profiles for both small and large wavelengths and all wave steepness resemble wave profiles for surface piecing cylinders for steady Fr in calm water, as previously investigated using CFDShip-Iowa V6.2 [16] albeit for much larger $0.2 \leq \mathrm{Fr} \leq 1.24$. Ref. [16] uses experimental [17] and empirical [18] data to validate run-up height and depression depth. Bernoulli equation along the free surface from upstream to the cylinder stagnation point estimates the run-up height as

$$
\begin{aligned}
& \eta_{R U} / d=F r_{B}^{2} / 2, \\
& F r_{B}=\sqrt{\overline{V^{2}}} / \sqrt{g d} .
\end{aligned}
$$

$\operatorname{Fr}_{B}$ is based on the square root of the time mean of the total velocity vector $V(t)=\left(\bar{u}+u^{\prime}, \bar{w}+w^{\prime}\right)$ squared,

$$
\overline{V^{2}}=\bar{u}^{2}+\overline{u^{\prime 2}}+\overline{w^{\prime 2}}
$$

$\bar{u}$ is the wave-induced mean velocity, as used in defining Fr and Re shown in Table 2. $u$ ' and $w^{\prime}$ are the wave induced velocity oscillations. Figure 7 includes $\eta_{\mathrm{RU}}$ estimated using the simulation velocities on the free surface at $2 \lambda$ upstream of the cylinder. The estimated $\eta_{\mathrm{RU}}$ values using the simulation velocities and $u_{\mathrm{I}} / w_{\mathrm{I}}$ in Eq. (1) and (2) were compared with the experimental $\eta_{0}$. The error is $24 \%$ for the estimation from CFD simulation velocities and $62 \%$ for $u_{\mathrm{I}} / w_{\mathrm{I}}$, respectively. Figure 8 shows $\bar{u}$ along vertical locations in water at $2 \lambda$ upstream of the cylinder. While steady current is constant at any depth, $\bar{u}$ is a function of vertical location $z$. The decaying rate is approximately proportional to $e^{15 \mathrm{kz}}$ and $e^{18 \mathrm{kz}}$ for short and long wave respectively, which is much faster than the decaying rates $e^{\mathrm{kz}}$ for $1^{\text {st }}$ order velocity component.

Figure 9(a) shows the comparisons for $+/-1^{\text {st }}$ harmonics of wave elevation and wave elevations at the crest $\bar{\eta}_{c} /$ trough $\bar{\eta}_{t}$ for $\lambda / d=4.7$ and $H / \lambda=1 / 30,1 / 16$ and $1 / 10$ conditions. Compared to the experimental results, V4.5 and V6.2 shows good accuracy in value and trend. In the results of V4.5, $1^{\text {st }}$ harmonics values at upstream are already more than 1.5 and keep increasing up to 2.0 on the weather face $\left(\theta=180^{\circ}\right)$ 
for the steepest case. Along the shoulder and leeward side, $1^{\text {st }}$ harmonics decreases. After a depression point at $\theta=45^{\circ}$, the values increase at $\theta=0^{\circ}$ and remain almost same at downstream. The elevations at crest/trough are not symmetric with respect to $x$-axis for all cases and the asymmetry increases for steeper wave, which can be seen by comparing crest/trough to $+/-1^{\text {st }}$ harmonic values, respectively. Crest height is similar at upstream and downstream, but trough height is lower at upstream than at downstream. On the weather face $\theta=180^{\circ}$, crest height is the highest $\bar{\eta}_{c} / A_{l}=1.7 \sim 2.1$ and trough height is the lowest $\bar{\eta}_{t} / A_{l} \approx-1.5$. While trough height increases along the shoulder and leeward face, crest height shows a depression on the leeward side $\theta=45^{\circ}$.

Figure 9 (b) shows comparisons of $+/-1^{\text {st }}$ harmonics of wave elevation and wave elevations at the crest $\bar{\eta}_{c} /$ trough $\bar{\eta}_{t}$ for $\lambda / d=21.9$ and $H / \lambda=1 / 30,1 / 16$ and $1 / 10$ conditions. V4.5 shows good agreement with the experiment data, even though the crest height on the weather face is over-estimated. Compared to short wave cases, $1^{\text {st }}$ harmonic values are nearly level with the incident wave amplitude, indicating small diffraction by the cylinder. The elevations at crest/trough are also asymmetric with respect to $x$-axis for all cases. The profiles of crest and trough heights are level, compared to short wave cases. At upstream and downstream, crest/trough heights are almost same. On weather face, crest height is the highest $\bar{\eta}_{c} / A_{l}=1.2 \sim 1.7$ at $\theta=180^{\circ}$ and trough height is the lowest $\bar{\eta}_{t} / A_{l} \approx-1.1$ at $\theta=135^{\circ}$. On leeward side, crest/trough heights are similar for all cases.

The $1^{\text {st }}$ harmonic wave profiles are similar in appearance to those for the mean wave profiles. The $1^{\text {st }}$ harmonic wave phases are shown in Table 5. The average errors for phases are $4.2 \%$ and $2.3 \%$ for short and long wave, respectively. For short waves, the $1^{\text {st }}$ harmonic wave phase shows small lead $\left(\approx 10^{\circ}\right)$ on the cylinder face and large lag $\left(\approx 120^{\circ}\right)$ on the cylinder back, whereas for long waves the $1^{\text {st }}$ harmonic wave phase shows fairly large nearly constant lead $\left(\approx 40 \sim 70^{\circ}\right)$.

Figure 10(a) shows comparisons of the $2^{\text {nd }}$ harmonics of wave elevation for $\lambda / d=4.7$ and $H / \lambda=1 / 30$, 1/16 and 1/10 conditions. V4.5 and V6.2 show reasonably accurate agreement with the experiment except for the case of $H / \lambda=1 / 10$. In the results of V4.5, the $2^{\text {nd }}$ harmonic values at downstream are higher than 
those at upstream, which indicates stronger non-linearity behind the cylinder. On the cylinder surface at $\theta=45^{\circ}$ and $135^{\circ}$, two depressions are shown. In contrast with mean and $1^{\text {st }}$ harmonics, $2^{\text {nd }}$ harmonic value is the highest on the shoulder of the cylinder $\left(\theta=90^{\circ}\right)$ and the highest value increases with increasing wave steepness $\left(\eta_{2} / A_{1}=0.2 \sim 0.4\right)$.

Figure 10(b) shows comparisons of the $2^{\text {nd }}$ harmonics of wave elevation for $\lambda / d=21.9$ and $H / \lambda=1 / 30$, $1 / 16$ and $1 / 10$ conditions. Compared to the experiment, results of V4.5 are reasonably accurate, but show some discrepancies for $H / \lambda=1 / 10$ case. In contrast to short wave case, the $2^{\text {nd }}$ harmonic values of V4.5 is higher at upstream than at downstream, indicating stronger non-linearity in front of the cylinder. On the weather side $\left(\theta=180^{\circ}\right), 2^{\text {nd }}$ harmonic value is the highest and increases for steeper wave condition. Along the shoulder, $2^{\text {nd }}$ harmonic value decreases and shows a depression point.

Validation study shows harmonics of wave elevations $\eta_{0}, \eta_{1}, \eta_{2}$ are generally in the range of $-0.1 \leq$ $\eta_{0} / A_{1} \leq 0.3,0.5 \leq \eta_{1} / A_{1} \leq 2.0$ and $0.05 \leq \eta_{2} / A_{1} \leq 0.4$, respectively. $1^{\text {st }}$ harmonics shows dominant values which are almost one order higher than mean and $2^{\text {nd }}$ harmonics, but maximum values of mean and $2^{\text {nd }}$ harmonics reach up to $15 \%$ and $20 \%$ of $1^{\text {st }}$ harmonics respectively, indicating severe non-linearity effect for steep wave.

Table 5 summarizes the errors of wave elevation harmonics, $1^{\text {st }}$ harmonic wave phase and crest/trough heights for $\lambda / d=4.7, H / \lambda=1 / 30$ and $\lambda / d=21.9, H / \lambda=1 / 10$. For short wave case, the average $\mathrm{E}$ for the $1^{\text {st }}$ order amp/phase and the $2^{\text {nd }}$ order variables are $5.5 / 4.2$ and $92 \% \mathrm{D}$, respectively. The errors increase from weather to shoulder to leeward side and are larger for off-body locations compared to onbody locations. For long wave case, the average $\mathrm{E}$ for the $1^{\text {st }}$ order amp/phase and the $2^{\text {nd }}$ order variables are $5.0 / 2.3$ and $84 \% \mathrm{D}$, respectively. The errors at weather side are large compared to short wave case. As shown in Fig. 11, energy dissipation caused by wave breaking was not described by the single-phase LS method of V4.5, which in turn could be the reason for large errors at the weather side for long wave.

The errors and differences for V4.5 and V6.2 for $\lambda / d=4.7, H / \lambda=1 / 16$ are also compared in Table 5 . The errors for most of $1^{\text {st }}$ and $2^{\text {nd }}$ order variables are smaller for V6.2 than for V4.5, but they are 
comparable and agree well with experiment. The differences between V4.5 and V6.2 are larger for the $2^{\text {nd }}$ order than the $1^{\text {st }}$ order variables and also show larger differences for on-body locations than for off-body locations. Even though V6.2 used coarse spatial/temporal resolution, it predicted wave run-up accurately due to high order numerical method and sharp interface treatment. V6.2, however, needs more expensive CPU time per iteration for the high order method and VOF function evaluation. As a result, overall computing costs for V4.5 and V6.2 are comparable in present study.

Table 5 also includes the averaged errors $\overline{\mathrm{E}}=\left(\mathrm{D}-\mathrm{S}_{\mathrm{OEC}}\right) \% \mathrm{D}$ where $\mathrm{D}$ and $\mathrm{S}_{\mathrm{OEC}}$ are the MOERI [9] wave elevation data and the other CFD data of ITTC workshop participants provided by OEC, which is 5.6\% and $141 \%$ for the $1^{\text {st }}$ and $2^{\text {nd }}$ order variables, respectively. Averaged $\mathrm{E}$ for all $1 \mathrm{C}$ cases are $5.8 \% \mathrm{D}$ and $67 \% \mathrm{D}$ for $1^{\text {st }}$ and $2^{\text {nd }}$ order variables, respectively, whereas $U_{F B}$ is $4.7 \%$ and $118 \%$. Thus, error for the $1^{\text {st }}$ order variables is slightly higher than $\mathrm{U}_{\mathrm{FB}}$ and $\overline{\mathrm{E}}$ but still comparable. The error for the $2^{\text {nd }}$ order variables is less than half of $\overline{\mathrm{E}}$.

Figure 11 shows photograph of experiment and instantaneous V4.5 wave pattern for $\lambda / d=21.9$, $H / \lambda=1 / 10$ case. The experiment exhibits wave breaking and spray on the weather and shoulder sides of the cylinder and a broken unsteady wake with air entrainment on the leeward side of the cylinder and wake. The V4.5 wave pattern is qualitatively similar but does not capture wave breaking/bubbly wake flow. Figure 12 shows comparison of wave pattern of V4.5 and V6.2 for $\lambda / d=4.7, H / \lambda=1 / 10$ case. Even though experimental photograph is not available, the comparison confirms that V6.2 has capability to describe wave breaking/spraying even with coarse grid resolution compared to V4.5 simulation.

\subsection{Four circular cylinders (4C)}

The errors of wave elevation harmonics, $1^{\text {st }}$ harmonic wave phase and crest/trough heights for $4 \mathrm{C}$, $\lambda / d=4.7, H / \lambda=1 / 30$ case are summarized in Table 5 . The $1^{\text {st }}$ harmonic wave phases show lead/lag with respect to the wave phase for short/long waves. The errors do not show clear trend regarding weather/shoulder faces of the cylinder, but shows that errors for crest/trough heights at the weather side 
are relatively high even under short wave condition. Since wave gages are located around the downwave cylinder, the interaction between cylinders could be related with the deterioration of accuracy at the weather side. Average $\mathrm{E}$ for $\eta_{1}$ is relatively low at the weather side, which suggests that majority of the errors for crest/trough heights at the weather side are not caused by the $1^{\text {st }}$ harmonics, but by the $2^{\text {nd }}$ or higher harmonics. In most cases, the errors for on-body locations are higher than for off-body locations. For $4 \mathrm{C}$ case, the average $\mathrm{E}$ for the $1^{\text {st }}$ and $2^{\text {nd }}$ order terms are $7.4 \%$ and $118 \%$, which seems reasonably accurate compared to $1 \mathrm{C}$ case, even if $\mathrm{U}_{\mathrm{FB}}$ and $\overline{\mathrm{E}}$ for $4 \mathrm{C}$ are not available.

\section{Discussion}

Stansberg and Kristiansen [5] categorized different contributions of non-linear effect to total wave amplification as follows

(i) $2^{\text {nd }}$-and higher-order components in the incident wave

(ii) $2^{\text {nd }}$ - and higher-order components due to interaction with the column

(iii) viscous effects ('blocking' due to flow separation)

(iv) local dissipation and breaking due to interaction with the columns

(v) thin 'jets' along columns

The non-linear contribution from factor (i) can be approximately up to $20 \%$ and is established well for regular undisturbed wave [5] for which the present results are consistent, as discussed in Section 6.1. The contribution from factor (ii) is discussed in terms of wave diffraction analysis in Section 6.2 and then interaction between cylinders is studied in Section 6.3, comparing the results of 1C and 4C. In Section 6.4, factor (iii) is also studied in terms of vortex structure. CFDShip-Iowa V6.2 is capable of studying factor (v), but grids with billions of points are required. 


\subsection{Effect of wave steepness and wave length}

According to linear potential theory, the incident wave is not distorted for long wave $(\lambda / d>15.7 \sim 20.9)$, while for short wave (approximately $\lambda / d<3.9 \sim 5.2$ ), the amplification factor is higher than 1.6. When a linear wave reflects perfectly from a vertical wall, it approaches to asymptotic value of 2.0. For short $\lambda / d=4.7$ waves, normalized wave crests are higher than 1.6 at weather side $\theta=180^{\circ}$. The steepest wave $H / \lambda=1 / 10$ produces even higher crest than 2.0 at $r=0.513 d, \theta=180^{\circ}$. The amplifications in long wave cases $\lambda / d=21.9$ are much less than those in short wave cases, but still exceeds 1.2 even for $H / \lambda=1 / 30$. In Figure 4 and 5, horizontal force is dominant in short wave case, while both of horizontal and vertical forces are important in long wave case, which confirms that wave length is major parameter for wave induced loads compared to wave steepness.

The harmonic analysis of wave run-up for present results confirms about 20-30\% of non-linearity for the steepest waves. Figure 13 shows mean, $1^{\text {st }}$ and $2^{\text {nd }}$ harmonics of wave elevations for short and long wave. The harmonic values were normalized by $1^{\text {st }}$ order wave amplitude $A_{l}$ in order to investigate nonlinear effect according to wave steepness. In terms of the normalized harmonic values, variation amount according to wave steepness indicates the effect of wave steepness. For linear wave with small wave steepness, mean and $2^{\text {nd }}$ harmonics are almost zero and $1^{\text {st }}$ harmonics are almost constant.

Observations from Fig. 13 can be organized into four categories: (1) upstream vs. downstream, (2) weather side, (3) leeward side, (4) shoulder.

(1) at upstream, harmonic values are almost same for short wave case, but difference between the harmonic values increases for long wave. At downstream, on the other hand, $1^{\text {st }}$ and $2^{\text {nd }}$ harmonics shows similar values for both short and long wave case.

(2) at weather side $\left(\theta=180^{\circ}\right.$ and $\left.135^{\circ}\right), 1^{\text {st }}$ and $2^{\text {nd }}$ harmonics for short wave increase with steepness, which is especially noticeable for the steepest wave. For long wave case, $1^{\text {st }}$ harmonics shows similar values, but mean and $2^{\text {nd }}$ harmonic values substantially increase with increasing steepness suggesting non-linear effect. 
(3) at leeward side $\left(\theta=45^{\circ}\right.$ and $\left.0^{\circ}\right), 1^{\text {st }}$ harmonics for short wave decrease with increasing steepness, but $2^{\text {nd }}$ harmonic values increase with steepness. Long wave case also shows similar trend.

(4) at shoulder $\left(\theta=90^{\circ}\right)$, mean and $1^{\text {st }}$ harmonic values for short wave decrease slightly, but $2^{\text {nd }}$ harmonics increase with increasing steepness. For long wave case, on the other hand, $1^{\text {st }}$ and $2^{\text {nd }}$ harmonics shows almost same for different wave steepness, but mean values increase with increasing steepness.

Based on the above, the $2^{\text {nd }}$ to $1^{\text {st }}$ harmonic ratio on the weather side decreases with steepness for short waves, but increases for long waves. For both of wave lengths, the $2^{\text {nd }}$ to $1^{\text {st }}$ harmonic ratio at leeward side increases by increasing steepness. At shoulder, the $2^{\text {nd }}$ to $1^{\text {st }}$ harmonic ratio increases with steepness for short wave, but remains almost constant for long wave.

\subsection{Wave diffraction analysis}

In order to study the sources of the non-linearity in total wave elevation, wave diffraction analysis was performed. As already mentioned in Section 3.2, the incident wave profile $\eta_{I}$ given in Eq. (1) or Eq. (2) can be different with those inside the domain of actual CFD simulation due to strong non-linear and viscous effect. Thus, we performed additional CFD simulations with no cylinder(s) to obtain CFD wave elevations without cylinder diffraction, which is denoted by $\eta_{N C}$. With $\eta_{N C}$, diffracted wave elevation $\left(\eta_{D}\right)$ is defined as:

$$
\eta_{D}=\eta_{T}-\eta_{N C}
$$

where $\eta_{T}$ is total wave elevation. The harmonics of the diffracted wave elevation $\eta_{D 0}, \eta_{D 1}, \eta_{D 2}$ was obtained using FFT.

Harmonic contours of diffracted wave for $1 \mathrm{C}$ short wave $(\lambda / d=4.7)$ are presented in Fig. 14. For $H / \lambda=1 / 30$ case, mean values are higher than calm water level right in front of the cylinder, indicating runup in mean wave profile. At upstream, mean values are partially higher and lower than calm water level 
but they converge to calm water level asymptotically. The $1^{\text {st }}$ harmonic component spreads toward upstream widely, but propagates along relatively narrow path behind the cylinder. The $2^{\text {nd }}$ harmonics shows spreading pattern like bird's footprint behind the cylinder. Meanwhile, the $3^{\text {rd }}$ harmonics is mostly observed in the vicinity of the cylinder.

For steeper wave condition $H / \lambda=1 / 10$, mean and $1^{\text {st }}$ harmonic values have similar pattern to the case of $H / \lambda=1 / 30$, but stronger diffractions are observed in wide region around the cylinder. The pattern of $2^{\text {nd }}$ harmonics is also similar to $H / \lambda=1 / 30$ case, but it spreads further with strong magnitude behind the cylinder. For the $3^{\text {rd }}$ harmonics, diffraction waves with stronger pattern are observed in much wider region behind the cylinder and the pattern is similar to that of $2^{\text {nd }}$ harmonics. The spreading pattern of diffraction wave confirms the observation previously in Section 6.1 that non-linearity for short wave decreases at weather side, but increases at leeward side by increasing steepness.

Harmonic contours for $1 \mathrm{C}$ long wave $(\lambda / d=21.9)$ were obtained, but the cylinder does not diffract the wave field compared to the short wave case. Especially for $H / \lambda=1 / 30$ case, $1^{\text {st }}$ harmonics doesn't show clear pattern for diffraction wave. The $2^{\text {nd }}$ and $3^{\text {rd }}$ harmonics spread toward upstream, but the diffraction strength is weak. For $H / \lambda=1 / 10$ case, diffraction strength becomes stronger, but strong diffraction is mainly staying near the cylinder, even though $2^{\text {nd }}$ and $3^{\text {rd }}$ harmonics show mild diffraction toward upstream.

Mean wave elevation contours in Fig. 14 are similar to those from experiment and CFD simulation for a vertical cylinder in steady current [19]. For present case, however, Kevin wave pattern behind the cylinder is less clear and diffraction from the cylinder spreads further towards upstream, which is attributed to high $\mathrm{Re}$ and low $\mathrm{Fr}$ compared to $\mathrm{Re}=2.7 \times 10^{4}$ and $\mathrm{Fr}=0.8$ in [19]. Compared to diffraction pattern for ship in head waves [20] [21], $1^{\text {st }}$ harmonics of diffracted wave in Fig. 14 shows significantly wider spreading pattern toward upstream. In addition, $2^{\text {nd }}$ harmonics behind the cylinder is also much clearer than that behind the ship in head waves. The most important factor causing the differences is steady horizontal velocity generated by towing the ship. In [20] and [21], wave induced current is less than $1 \%$ of the steady horizontal velocity and thus the diffraction pattern is affected mainly by the steady 
horizontal velocity. In addition, for present study, wave steepness is much higher and cylinder has blunt geometry rather than sharp shape for ship, which leads quite different diffraction pattern from that for ship in head waves.

The highest values in Fig. 14 and the ratios of them to $1^{\text {st }}$ harmonics were investigated, which shows that the normalized $1^{\text {st }}$ harmonics of diffracted wave reaches up to 1.01 . The $2^{\text {nd }}$ and $3^{\text {rd }}$ harmonics have much smaller magnitude than that of $1^{\text {st }}$ harmonics, but still exert their influences on the diffraction. Especially, $2^{\text {nd }}$ harmonics for short wave is $18 \%$ of $1^{\text {st }}$ harmonics for $H / \lambda=1 / 30$ and increases up to $41 \%$ by steepness increase. The $3^{\text {rd }}$ to $1^{\text {st }}$ harmonic ratio also increases from $8.8 \%$ to $16 \%$ by steepness increase, suggesting strong non-linearity. For long wave case, the $2^{\text {nd }}$ to $1^{\text {st }}$ harmonic ratio is $14 \%$ for $H / \lambda=1 / 30$ and increases up to $34 \%$ for $H / \lambda=1 / 10$. The $3^{\text {rd }}$ to $1^{\text {st }}$ harmonic ratio also increases from $9.1 \%$ to $20 \%$ by steepness increase. Nevertheless, diffraction pattern for long wave was observed only near the cylinder.

Figure 15 shows harmonic contours of diffracted wave for $4 \mathrm{C}$. The incident wave condition is $\lambda / d=4.7$ and $H / \lambda=1 / 30$, which is same with the case of Fig. 14(a). The pattern of mean and the $3^{\text {rd }}$ harmonics seems like superposition of diffractions from each cylinder, which is especially true around two upwave cylinders. The $2^{\text {nd }}$ harmonics shows quite complicated pattern with increased magnitude, which is considered due to strong interaction between diffracted waves. Moreover, the $1^{\text {st }}$ harmonic diffraction is distributed at the center of the four cylinders as well as in-between upwave and downwave cylinders, which can be also considered as interaction effect. Even if the highest $1^{\text {st }}$ harmonic values are almost same for $1 \mathrm{C}$ and $4 \mathrm{C}$, the $2^{\text {nd }}$ to $1^{\text {st }}$ harmonic ratio is increased and reaches up to $25 \%$. The $3^{\text {rd }}$ to $1^{\text {st }}$ harmonic ratio is also increased to $12 \%$.

\subsection{Interaction between cylinders}

As observed in previous subsection, the magnitude and spreading pattern of diffracted waves are affected by interaction between cylinders. Wave elevations at crest/trough are compared in Table 6 for 1C and $4 \mathrm{C}$, which also includes the experimental results $\triangle \mathrm{EFD}_{4 \mathrm{C}} \% \mathrm{EFD}_{1 \mathrm{C}}$ (or $\triangle \mathrm{CFD}_{4 \mathrm{C}} \% \mathrm{CFD} \mathrm{DC}_{1 \mathrm{C}}$ ). The difference is caused only by interaction between cylinders, since the other conditions including incident 
wave, grid resolution etc. are controlled. For crest height, the averaged difference between experiment data for $1 \mathrm{C}$ and $4 \mathrm{C}\left(\triangle \mathrm{EFD}_{4 \mathrm{C}} \% \mathrm{EFD}_{1 \mathrm{C}}\right)$ increases with increasing $\theta(8.82 \% \rightarrow 13.39 \% \rightarrow 25.26 \%)$ and CFD result also shows the same tendency $(5.25 \% \rightarrow 12.69 \% \rightarrow 17.34 \%)$. For trough height, the average of $\triangle \mathrm{EFD}_{4 \mathrm{C}} \% \mathrm{EFD}_{1 \mathrm{C}}$ decreases with increasing $\theta(16.93 \% \rightarrow 7.21 \% \rightarrow 5.81 \%)$, but CFD does not capture the tendency $(4.32 \% \rightarrow 13.34 \% \rightarrow 10.46 \%)$. The interaction effect on the crest height is stronger at shoulder $\left(\theta=270^{\circ}\right)$ than at weather side $\left(\theta=180^{\circ}\right)$. On the other hand, the trough height is more affected by the interaction at weather side $\left(\theta=180^{\circ}\right)$ than at shoulder $\left(\theta=270^{\circ}\right)$.

A distinct feature of wave run-up for $4 \mathrm{C}$ is near-trapping phenomenon [22]. When four cylinders are located at the corners of a square, near-trapping can be one of the main contributing factors which give rise to high localized wave elevations inside the square region. Although near-trapping seems to make a partial effect on the crest height at shoulder, intensive near-trapping has not been observed in present test case. It is thought that the less sheltered geometry $\left(\beta=0^{\circ}\right)$ and much lower $d / l$ parameter prevent neartrapping for present test [22]. On the other hand, in the case with $\beta=45^{\circ}$, even with same $d / l$ parameter and incident wave, near-trapping condition $(\lambda \approx \sqrt{2} l-d)[23]$ is almost satisfied, which motivates future works to investigate near-trapping phenomenon in detail.

\subsection{Vortex shedding}

The relevant variables for analysis of vortex shedding are the number of shedding cycles $T_{k}$ per wave half period $(T / 2) / T_{k}$, where $T_{k}=1 / f_{k}$ and $f_{k}$ is the shedding frequency, and Keulegan-Carpenter number $K_{c}=u_{\zeta} \cdot T / d$, where $u_{\zeta}$ is the maximum velocity $\left(u_{\zeta}=\bar{u}+\left|u^{\prime}\right|_{\max }\right)$ evaluated on free surface $z=\zeta$ at $x=2 \lambda$ upstream of the cylinder. $f_{k}$ can be estimated using single phase cylinder Strouhal

number $S t=\frac{f_{k} d}{u_{\zeta}}=\mathrm{S}(\mathrm{Re}) \sim 0.25$ for post critical Re. Table 7 shows $(T / 2) / T_{k}$ and $K_{c}$ values. It also shows $A_{l} / T_{d}, \lambda / T_{d}, u_{\mathrm{T}_{\mathrm{d}}}$ and $u_{\mathrm{T}_{\mathrm{d}}} / u_{\zeta}$ values where $u_{\mathrm{T}_{\mathrm{d}}}$ is the maximum velocity at $z=T_{d}$ at $x=2 \lambda$ upstream of the cylinder. $A_{l} / T_{d}$ values are large and increase with wave steepness, reaching up to 0.7 for steepest long wave case. $\lambda / T_{d}$ also shows large values, especially for long wave cases. $u_{\zeta}$ and $u_{\mathrm{T}_{\mathrm{d}}}$ increase 
with increasing wave steepness/wave length and $u_{\mathrm{T}_{\mathrm{d}}} / u_{\zeta}$ shows that velocity decays 4.6 times faster over depth for short waves than for long waves. $(T / 2) / T_{k}$ values are less than 0.18 for short waves, but reaches up to 0.83 for long wave, which indicates insufficient time to develop vortex shedding for short wave cases. $K_{c}$ values are less than 1.5 for short wave, but the highest $\mathrm{K}_{\mathrm{c}}$ reaches up to 6.9 for long wave. Sarpkaya [24] used various ranges of $\mathrm{K}_{\mathrm{c}}$ for harmonically oscillating single phase flows to describe the evolution of vortices: (a) $\mathrm{K}_{\mathrm{c}}<4$; no vortex, (b) $4<\mathrm{K}_{\mathrm{c}}<8$; symmetric vortex shedding, (c) $\mathrm{K}_{\mathrm{c}}>8$; asymmetric vortex shedding. In case of incoming wave for surface-piercing cylinder, $\mathrm{K}_{\mathrm{c}} \approx 6$ was considered as a criterion for onset of separated vortices by Mei [25], but it has been argued that vortex shedding has also been observed at lower $\mathrm{K}_{\mathrm{c}}$ numbers [26].

Figure 16 and 17 show instantaneous air/water interface and Q-criterion contour for short and long wave with $H / \lambda=1 / 10$, respectively. Positive Q-criterion value indicates vortex. Small and weak vortices are shown even for $K_{c}=1.5$ (Fig. 16) and vortices grow in size and strength significantly for $K_{c}=6.9$ (Fig. 17). The structure of vortices doesn't show regular pattern such as periodic shedding. Figure 18 presents mean air/water interface and Q-criterion contour for short and long wave with $H / \lambda=1 / 10$. In Fig. 18(a), small vortices are mainly observed near air/water interface. Due to positive/negative horizontal velocity field of incident wave at crest/trough, vortices are generated at upstream as well as at downstream. In Fig. 18(b), relatively strong vortices are observed in wide region, but the structure of vortices still shows irregular pattern.

In Table $7, C_{d}$ and $C_{m}$ values are also presented. By using the time histories of horizontal force, horizontal velocity and its time derivative extracted from CFD simulation, $C_{d}$ and $C_{m}$ can be calculated through Morison equation and have been compared with those in the study of Sarpkaya [24]. $C_{d} / C_{m}$ for CFD results are $0.33 / 1.3$ and $0.42 / 1.3$ for short and long wave respectively. $T$ he $C_{d} / C_{m}$ values for short and long wave are 49\% / 26\% and 35\% / 26\% lower than Sarpkaya's data for the corresponding Reynolds number, respectively, even if $\mathrm{K}_{\mathrm{c}}$ for present study is in the much lower range. It should be noted that Sarpkaya's data is for two-dimensional harmonic single phase flow generated by harmonically 
oscillating cylinder in deep water, whereas present CFD handles surface-piercing truncated cylinder due to incoming wave. As mentioned by Sarpkaya [24], the differences seem to be caused by (a) vertical velocities, (b) vertical gradient of horizontal velocity amplitude, (c) non-linear harmonic component of the horizontal velocity and (d) free surface effect.

Based on parameters in Table 7 and Q-criterion contours, vortex shedding can be discussed in two perspectives: (1) size/strength of the vortices, (2) irregularity,

(1) Size/strength of the vortices are functions of $(T / 2) / T_{k}$ or $K_{c}$, where $(T / 2) / T_{k}=S t \cdot K_{c} / 2$ and St is almost constant for post critical Re. Short wave period reduces the size/strength of vortices by breaking them up into small and weak pieces.

(2) The vortices do not show regular structure such as periodic shedding. Incident wave keeps changing velocity direction for crest/ trough, which disturbs a development of regular pattern.

Therefore, for cases with same wave period, higher wave steepness produces higher $K_{c}$ values and in turn induces strong vortices.

\section{Conclusions}

The capabilities of CFDShip-Iowa V4.5 and V6.2 simulations for wave run-up for single/multiple surface-piercing cylinders in regular waves were assessed. The simulations were conducted for various wave conditions including $\lambda / d=4.7$ and $\lambda / d=21.9$ with $H / \lambda=1 / 30,1 / 16$ and $1 / 10$. Statistical convergence and grid, domain and turbulence model sensitivity were evaluated. The simulations were qualitatively validated using facility bias $\mathrm{U}_{\mathrm{FB}}$ and error $\mathrm{E}$ between the experiment and simulation. Variables were grouped into first $\left(1^{\text {st }}\right.$ harmonics) and second (mean and $2^{\text {nd }}$ harmonics) order terms for separate assessment capabilities of each both for the experiments and CFD. Relation between wave induced current and run-up height in mean wave profile was analyzed and the effect of wave steepness/wave length, wave diffraction, interaction among cylinders, and vortex shedding structure were discussed. 
Statistical convergence uncertainty $\mathrm{U}_{\mathrm{I}}$ values were less than $1 \%$ for both the experiment and simulation data. The grid, domain and turbulence model sensitivities were less than the respective $\mathrm{U}_{\mathrm{FB}}$ for $1^{\text {st }}$ and $2^{\text {nd }}$ order variables. The results indicated that both the large and medium domains should be sufficient for the validation studies. The turbulence model sensitivities were also small such that the URANS turbulence model was sufficient for the validation studies.

For horizontal/vertical forces, the validation studies showed that the errors E were 72 and $23 / 2 \% \mathrm{D}$ for $2^{\text {nd }}$ order and $1^{\text {st }}$ order amp/phase variables, respectively. For wave elevations, the detailed study of the wave run-up profile showed that the mean wave field elevations were similar to the free surface elevations for a cylinder in a steady current due to the large wave induced current (up to $18 \%$ of the horizontal velocity for the steepest wave). Investigation on wave induced current and run-up height in mean wave profile showed that the maximum run-up $\left(\eta_{\mathrm{RU}}\right)$ in mean wave elevation can be estimated accurately using Bernoulli equation. The maximum values of mean and $2^{\text {nd }}$ harmonics reached up to $15 \%$ and $20 \%$ of $1^{\text {st }}$ harmonics respectively, indicating severe non-linearity effect for steep wave. The error E for all $1 \mathrm{C}$ cases was $5.8 \% \mathrm{D} / 67 \% \mathrm{D}$ for $1^{\text {st }} / 2^{\text {nd }}$ order variables, respectively, whereas $\mathrm{U}_{\mathrm{FB}}$ was $4.7 \% / 118 \%$ and averaged errors $\overline{\mathrm{E}}$ for the other CFD data provided by OEC was 5.6\%/ 141\%. Thus, error for the $1^{\text {st }}$ order variables is slightly higher than $\mathrm{U}_{\mathrm{FB}}$ and $\overline{\mathrm{E}}$ but still comparable. The error for the $2^{\text {nd }}$ order variables is less than half of $\overline{\mathrm{E}}$. The average $\mathrm{E}$ of $4 \mathrm{C}$ for the $1^{\text {st }}$ and $2^{\text {nd }}$ order terms are $7.4 \%$ and $118 \%$, which seemed reasonably accurate compared to $1 \mathrm{C}$ case, even if $\mathrm{U}_{\mathrm{FB}}$ and $\overline{\mathrm{E}}$ for $4 \mathrm{C}$ were not available.

Comparison between V4.5 and V6.2 showed that results from both agreed well with experiment, even if V6.2 had slightly smaller error than V4.5 for most of $1^{\text {st }}$ and $2^{\text {nd }}$ order terms. The comparison also showed the merits of each version. V4.5 is a generalized/robust code to guarantee relatively easy application for complex/large-scale problem, whereas V6.2 has capability to describe more detailed wave breaking/spraying utilizing high order method and VOF sharp interface treatment.

For wave steepness/wave length effect, wave length was major factor for wave induced loads compared to wave steepness. The $2^{\text {nd }}$ to $1^{\text {st }}$ harmonic ratio on the weather side decreased with steepness for short waves, but increased for long waves. For both of wave lengths, the $2^{\text {nd }}$ to $1^{\text {st }}$ harmonic ratio at 
leeward side increased by increasing steepness. The wave diffraction analyses showed that $2^{\text {nd }} / 3^{\text {rd }}$ to $1^{\text {st }}$ harmonic ratio in diffracted wave contour were 18 41\% / 9 16\% for short wave, suggesting stronger non-linearity for steeper wave. Kevin wave in mean wave elevation contours was similar but less clear due to high Re and low Fr, compared to that for a vertical cylinder in steady current. Compared to diffraction pattern for ship in head waves, $1^{\text {st }}$ harmonics of diffracted wave showed significantly wider spreading pattern toward upstream due to different dominance of wave induced current, wave steepness and body geometry. The highest $1^{\text {st }}$ harmonic values were almost same for $1 \mathrm{C}$ and $4 \mathrm{C}$, but the $2^{\text {nd }} / 3^{\text {rd }}$ to $1^{\text {st }}$ harmonic ratio increased up to $25 \% / 12 \%$ for $4 \mathrm{C}$ case.

Comparison of wave elevations at crest/trough for $1 \mathrm{C}$ and $4 \mathrm{C}$ showed that the interaction of cylinders increased trough height for $4-10 \%$ whilst crest height increased about 5-17\%. The strongest interaction effect on the crest height was found at the shoulder side of the cylinders. Intensive near-trapping was not observed in present test case. Studies on vortex shedding showed that size/strength of the vortices were functions of $(T / 2) / T_{k}$ or $K_{c}$. Short wave case had insufficient time to develop vortex shedding, compared to long wave case. Regular structure such as periodic shedding was not observed since velocity direction change for wave crest/trough disturbed regular pattern developing, especially for low $K_{c}$ value.

\section{Acknowledgement}

This work was sponsored by the Office of Naval Research (ONR) under Grant No. N000141-01-001-7, with Dr. Ki-Han Kim as the program manager. The calculations presented in this paper were

performed at the Department of Defense (DoD) Supercomputing Resource Centers (Navy DSRC) through the High Performance Computing Modernization Program (HPCMP).

\section{References}

[1] McCamy R. and Fuchs R., "Wave forces on piles: a diffraction theory," in Tech. Memo No. 69. Beach Erosion Board, Washington, DC, 1954.

[2] Kim, M. H. and Yue, D. K. P., "The complete second-order diffraction solution for an axisymmetric body: Part 
1. Monochromatic incident waves," J. Fluid Mech., vol. 200, pp. 235-264, 1989.

[3] Kriebel, D. L., "Nonlinear wave interaction with a vertical circular cylinder. Part II. Wave run-up," Ocean Eng., vol. 19, p. 75-99, 1992.

[4] Stansberg, C. T. and Nielsen, F. G., "Nonlinear wave-structure interaction on floating production systems," in Proc. 11th ISOPE Conf., Stavanger, Norway, 2001.

[5] Stansberg, C. T. and Kristiansen, T., "Non-linear scattering of steep surface waves around vertical columns," Appl. Ocean Res., vol. 27, pp. 65-80, 2005.

[6] Danmeier, D. G., Seah, R. K. M., Finnigan, T., Roddier, D., Aubault, A., Vache, M., and Imaura, J. T., "Validation of wave run-up calculation methods for a gravity based structure," in Proc. ASME 27th Int. Conf. Offshore Mech. and Arct. Eng., Estoril, Portugal, 2008.

[7] Bøckmann, A., Pâkozdi, C., Kristiansen, T., Jang, H., and Kim, J., "An experimental and computational development of a benchmark solution for the validation of numerical wave tanks," in Proc. ASME 33rd Int. Conf. Ocean, Offshore and Arctic Eng., San Francisco, USA, 2014.

[8] Yoon, S.-H., Kim, D.-H., Hosseini, H., Yang, J. and Stern, F., "High-fidelity CFD simulation of wave run-up around vertical cylinders in monochromatic waves," in Proc. ITTC Workshop on Wave Run-up and Vortex Shedding, Nantes, France, 2013.

[9] Sung, H. G., Kim, Y. S., Nam, B. W., and Hong, S. Y., "Experimental investigation of wave loads on a truncated vertical circular cylinder," in Proc. Korean Society of Ocean Eng. (in Korean), Daejon, Korea, 2007.

[10] Stansberg, C. T., Baarholm, R., Kristiansen, T., Hansen, E. W. M. and Rortveit, G., "Extreme wave amplification and impact loads on offshore structures," in Offshore Technology Conference, 2005.

[11] Huang, J., Carrica, P., and Stern, F., "Semi-coupled air/water immersed boundary approach for curvilinear dynamic overset grids with application to ship hydrodynamics," Int. J. Numerical Methods in Fluids, vol. 58, pp. 591-624, 2008.

[12] Yang, J., Wang, Z., Yeon, S.-M., Koo, B., and Stern, F., "High-fidelity curvilinear-grid two-phase flow solvers for ship hydrodynamics," in 29th Symposium Naval Hydrodynamics, Gothenburg, Sweden, 2012.

[13] Stern, F., Wilson, R. V., Coleman, H. W., and Paterson, E. G., "Comprehensive approach to verification and validation of CFD simulations_-Part 1: Methodology and procedures," ASME J. Fluids Eng., vol. 123, p. 793$802,2001$.

[14] Fenton, J. D., "A fifth-order stokes theory for steady waves," J. of Waterway, Port, Coastal and Ocean Eng., vol. 111 , no. 2 , pp. 216-234, 1985.

[15] Yang, K.-K., Nam, B.-W., and Kim, Y., "Numerical simulation of 3D free-surface flows by using CIP-based and FV-based methods," Int. J. of Ocean System Eng., vol. 1, no. 3, pp. 136-143, 2011.

[16] Koo, B., "Numerical study of two-phase air-water interfacial flow: plunging wave breaking and vortexinterface interaction," Ph.D. Thesis, University of Iowa, 2011.

[17] Chaplin, J. R. and Teigen, P., "Steady flow past a vertical surface-piercing circular cylinder," J. of Fluids and Structures, vol. 18, pp. 271-285, 2003.

[18] Hay, A.D., "Flow about semi-submerged cylinders of finite length," Princeton University Report, NJ, 1947. 
[19] Suh, J., Yang, J. and Stern, F., "The effect of air-water interface on the vortex shedding from a vertical circular cylinder," J. of Fluids and Structures, vol. 27, no. 1, p. 1-22, 2011.

[20] Gui., L., Longo, L., Metcal, B., Shao, J., and Stern, F., "Forces, moment, and wave pattern for naval combatant in regular head waves-Part 2: Measurement results and discussions," Experiments in Fluids, vol. 32, no. 1, p. 27-36, 2002.

[21] Hosseini, H., Carrica, P., Kim, H., Toda, Y., and Stern, F., "CFD V\&V of added resistance of KVLCC2 with fixed and free surge conditions for short and long head waves," Ocean Engineering, vol. 59, p. 240-273, 2013.

[22] Ohl, C. O. G., Eatock Taylor, R., Taylor, P. H., and Borthwick, A. G. L., "Water wave diffraction by a cylinder array. Part 1. Regular waves," J. Fluid Mech., vol. 442, pp. 1-32, 2001.

[23] Contento, G., D’Este, F., Sicchiero, M., Codiglia, R., and Calzà, M., "Run-up and wave forces on an array of vertical circular cylinders: experimental study on second-order near trapping," Int. J. of Offshore and Polar Eng., vol. 15, no. 2, pp. 96-103, 2005.

[24] Sarpkaya, T. and Isaacson, M., Mechanics of wave forces on offshore structures, New: Van Nostrand Reinhold, 1981.

[25] Mei, C. C, The applied dynamics of ocean surface waves, Singapore: World Scientific, 1989.

[26] Trulsen, K., and Teigen, P., "Wave scattering around a vertical cylinder: fully nonlinear potential flow calculations compared with low order perturbation results and experiments," in Proc. 21st Int. Conf. On Offshore Mech. and Artic Eng., 2002. 


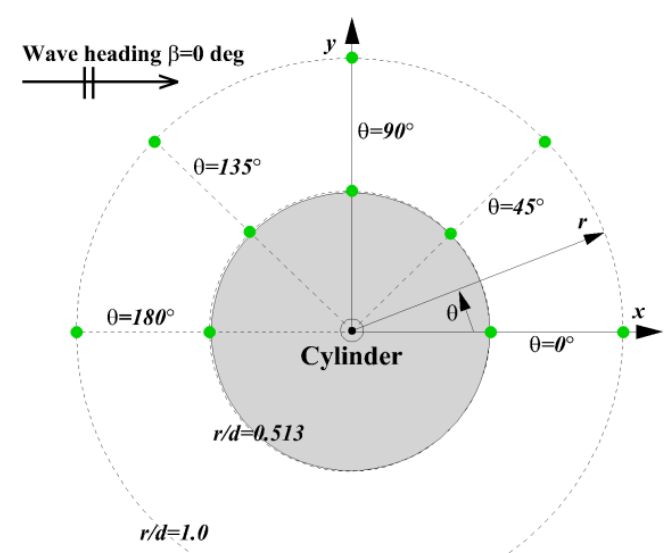

(a)

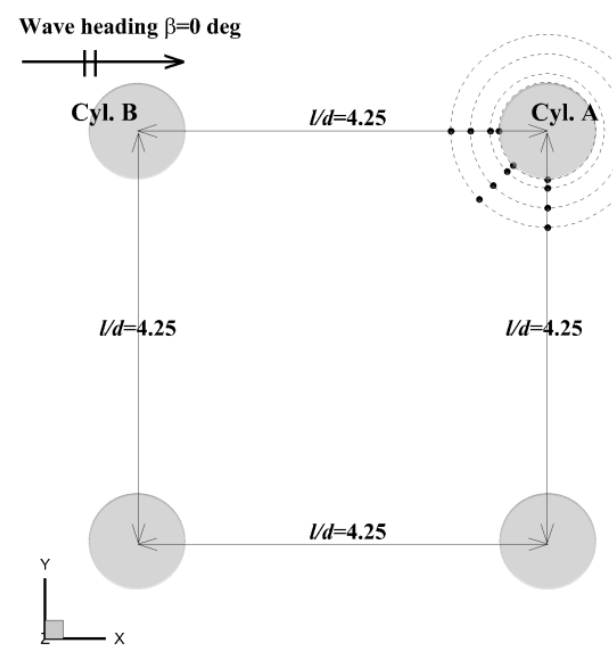

(b)

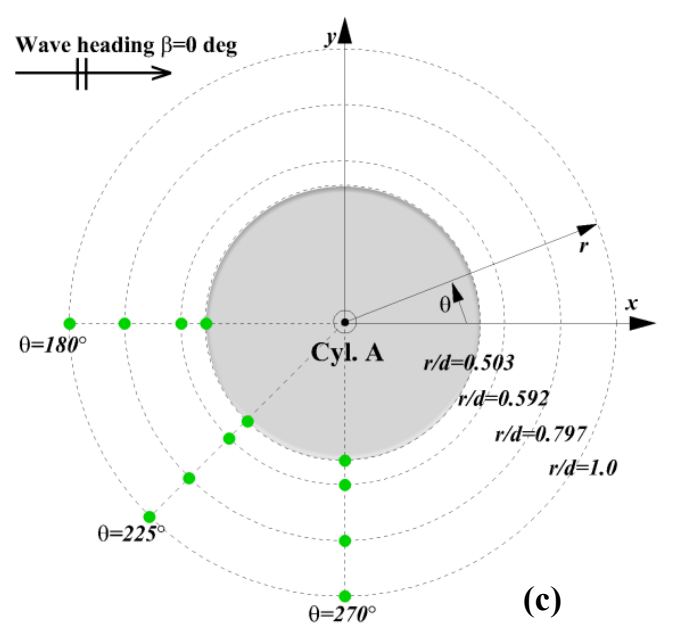

Figure 1 Locations of wave probes around circular cylinders: green circles $(\bullet)$ indicate wave probes, (a) single circular cylinder, (b) arrangement of four circular cylinders, (c) wave probes for four circular cylinders 

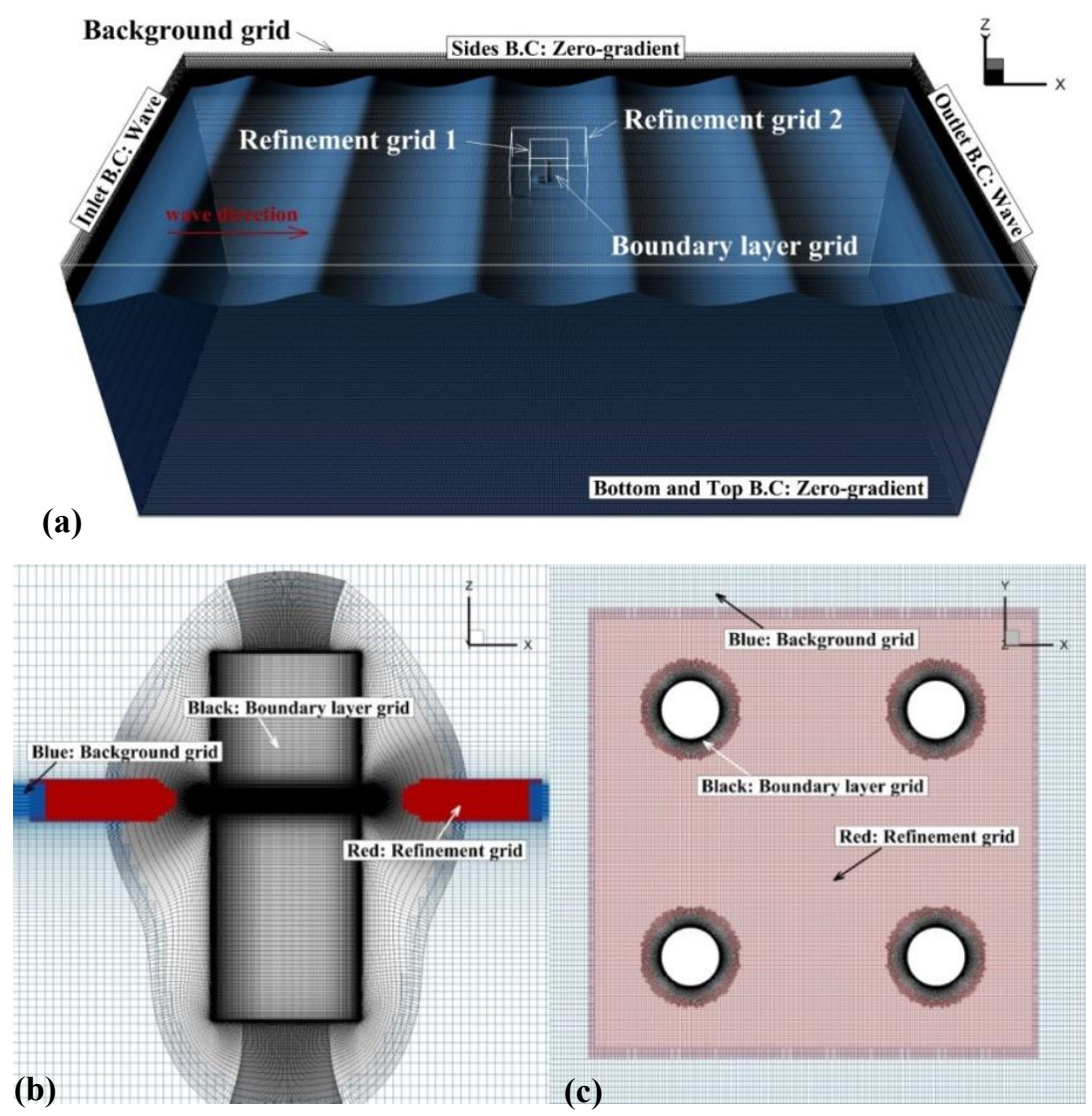

Figure 2 Overset grid system and boundary condition for V4.5 simulation: (a) computational domain and boundary conditions, (b) side view for 1C, (c) top view for 4C

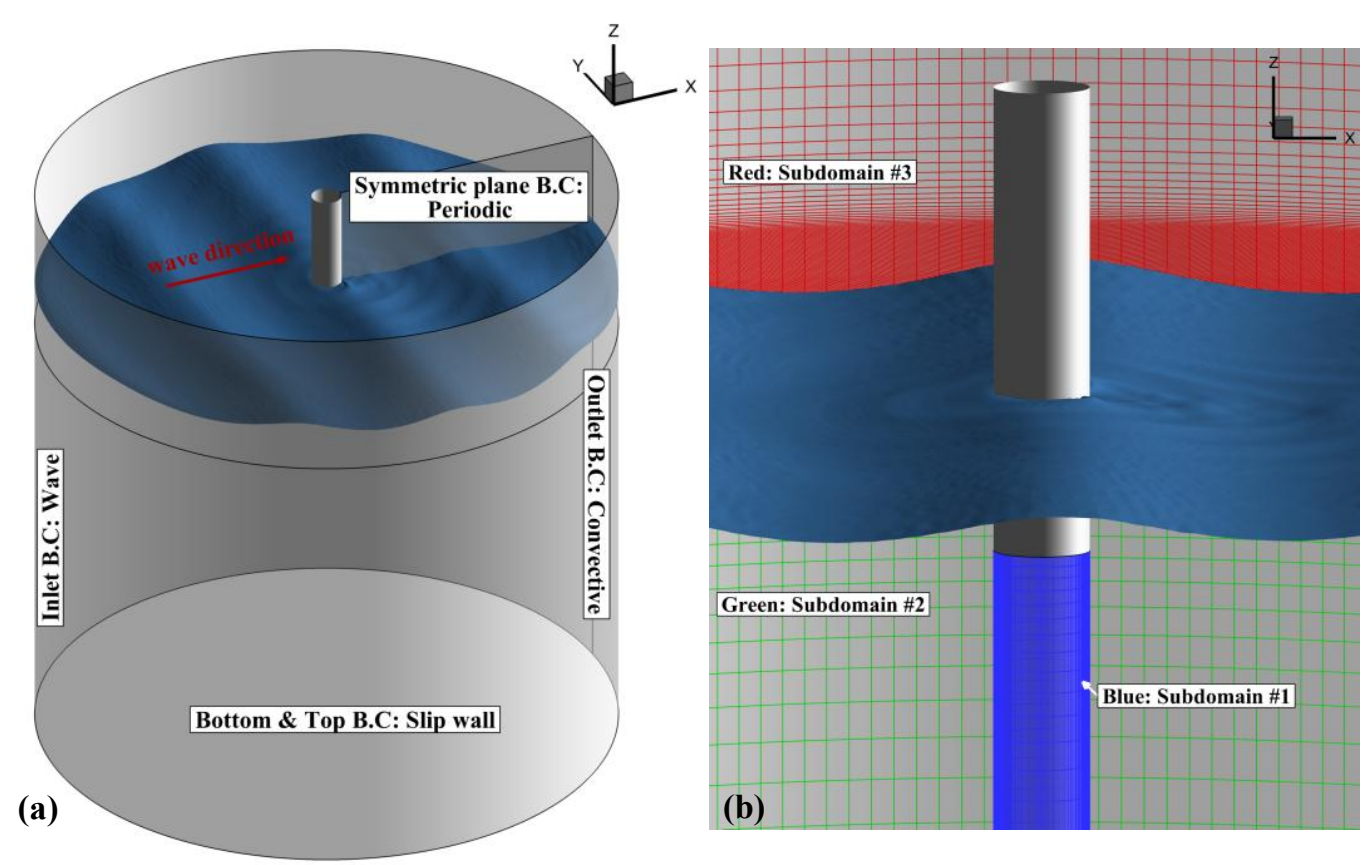

Figure 3 Cylindrical grid system and boundary condition for V6.2 simulation:

(a) computational domain and boundary conditions, (b) surface mesh 

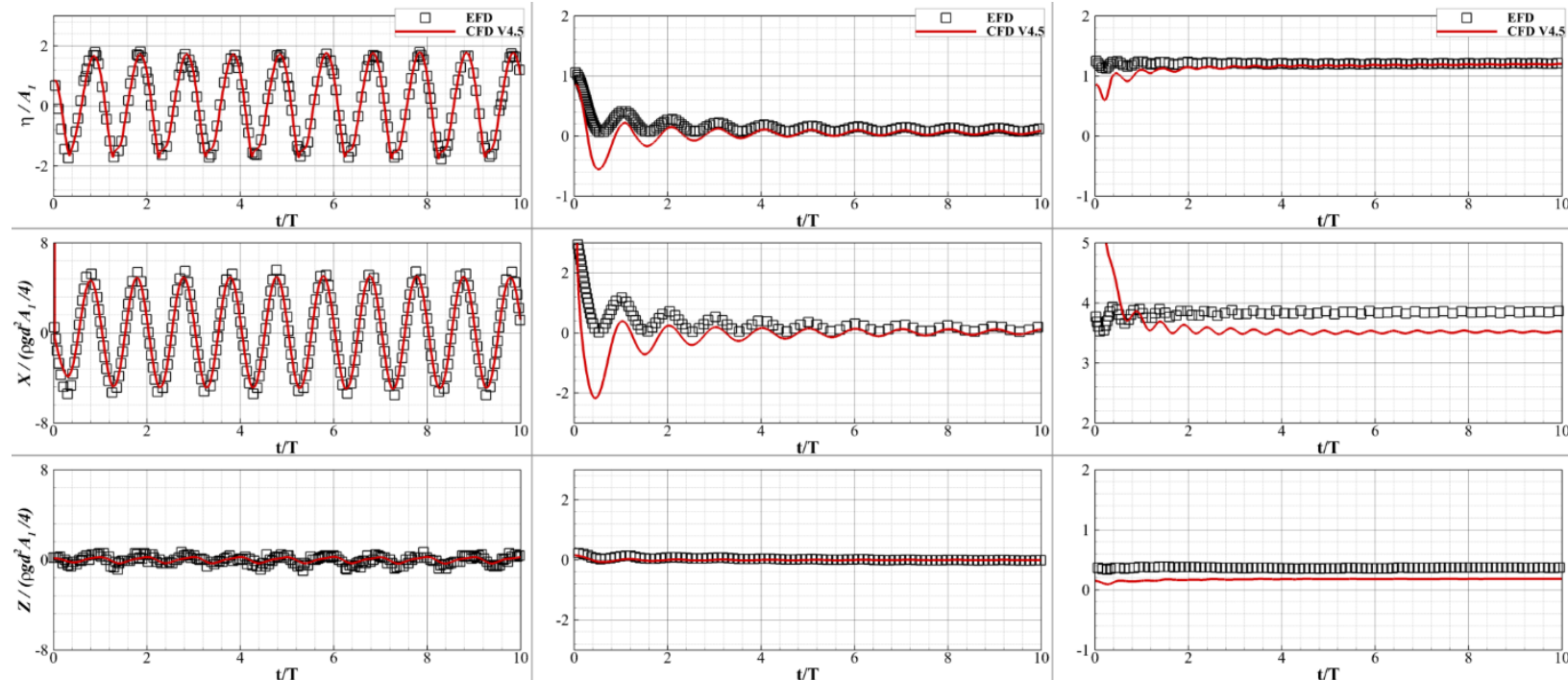

(a)

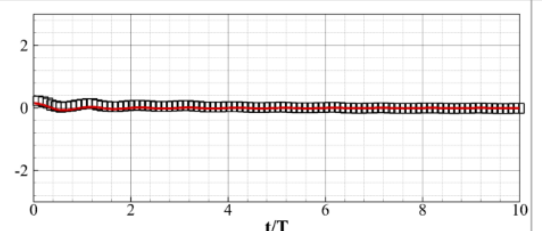

(b)

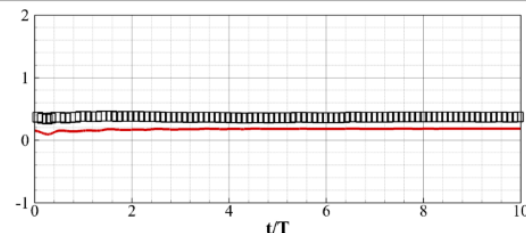

(c)

Figure 4 Time history results for $\lambda / d=4.7, H / \lambda=1 / 30$ (black symbol: EFD, red line: CFD V4.5):

(a) wave elevation at $r / d=0.513, \theta=180^{\circ}$ and forces (b) running mean, (c) running root mean square
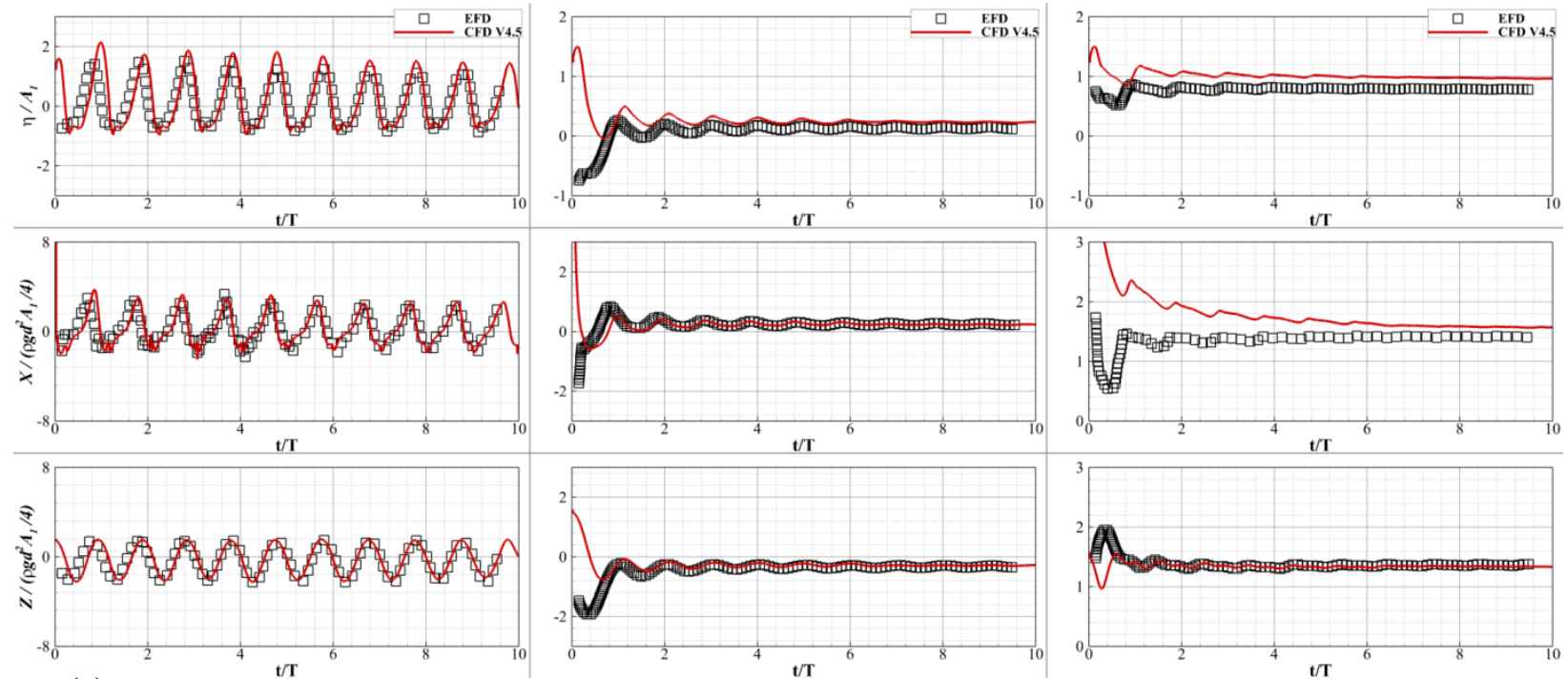

(b)

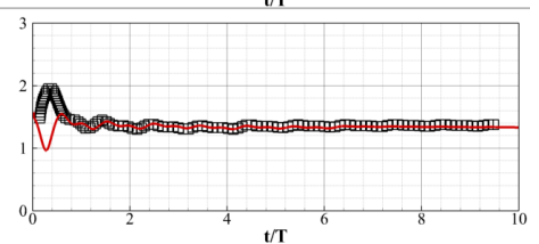

(c)

Figure 5 Time history results for $\lambda / d=21.9, H / \lambda=1 / 10$ (black symbol: EFD, red line: CFD V4.5): (a) wave elevation at $r / d=0.513, \theta=180^{\circ}$ and forces (b) running mean, (c) running root mean square 

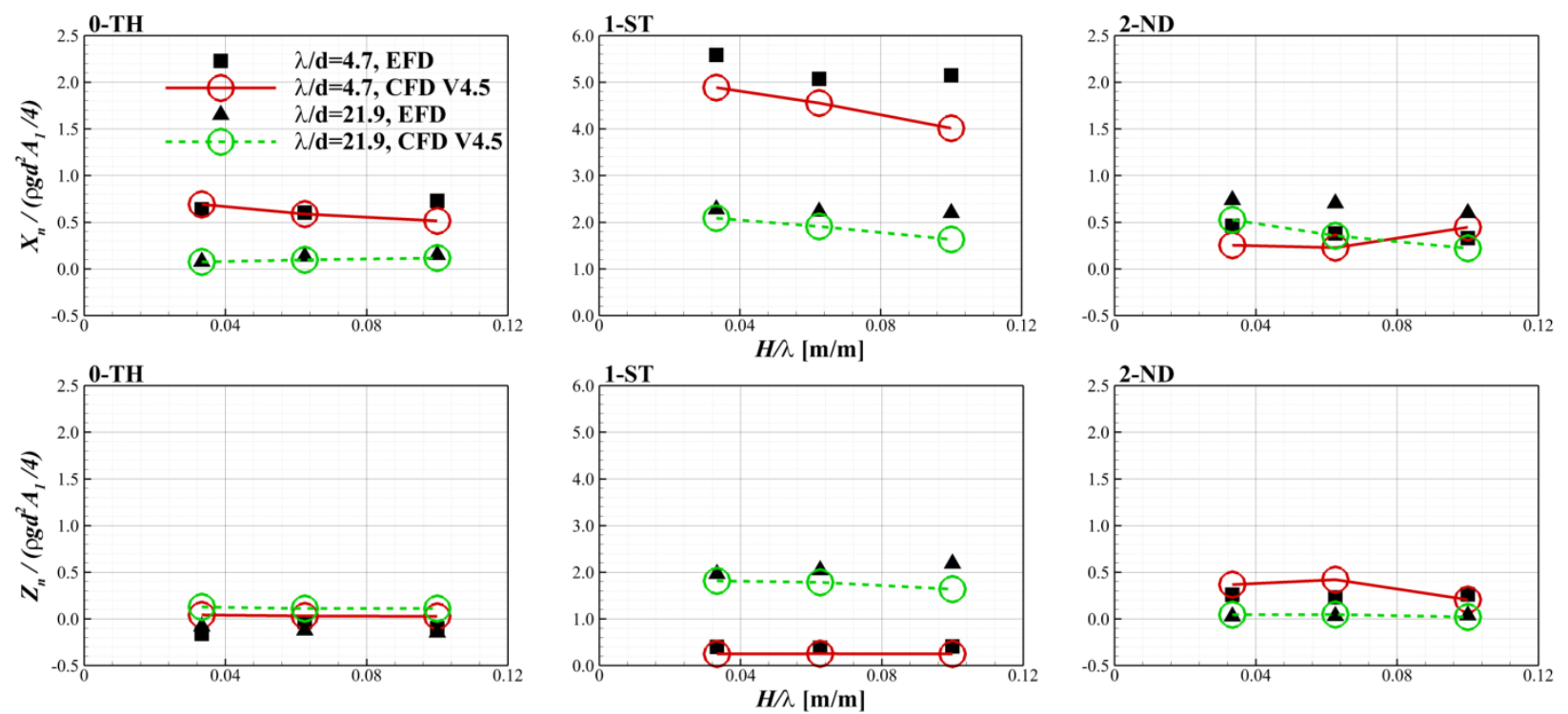

Figure 6 Mean, $1^{\text {st }}$ and $2^{\text {nd }}$ harmonics of horizontal and vertical forces (1C) 

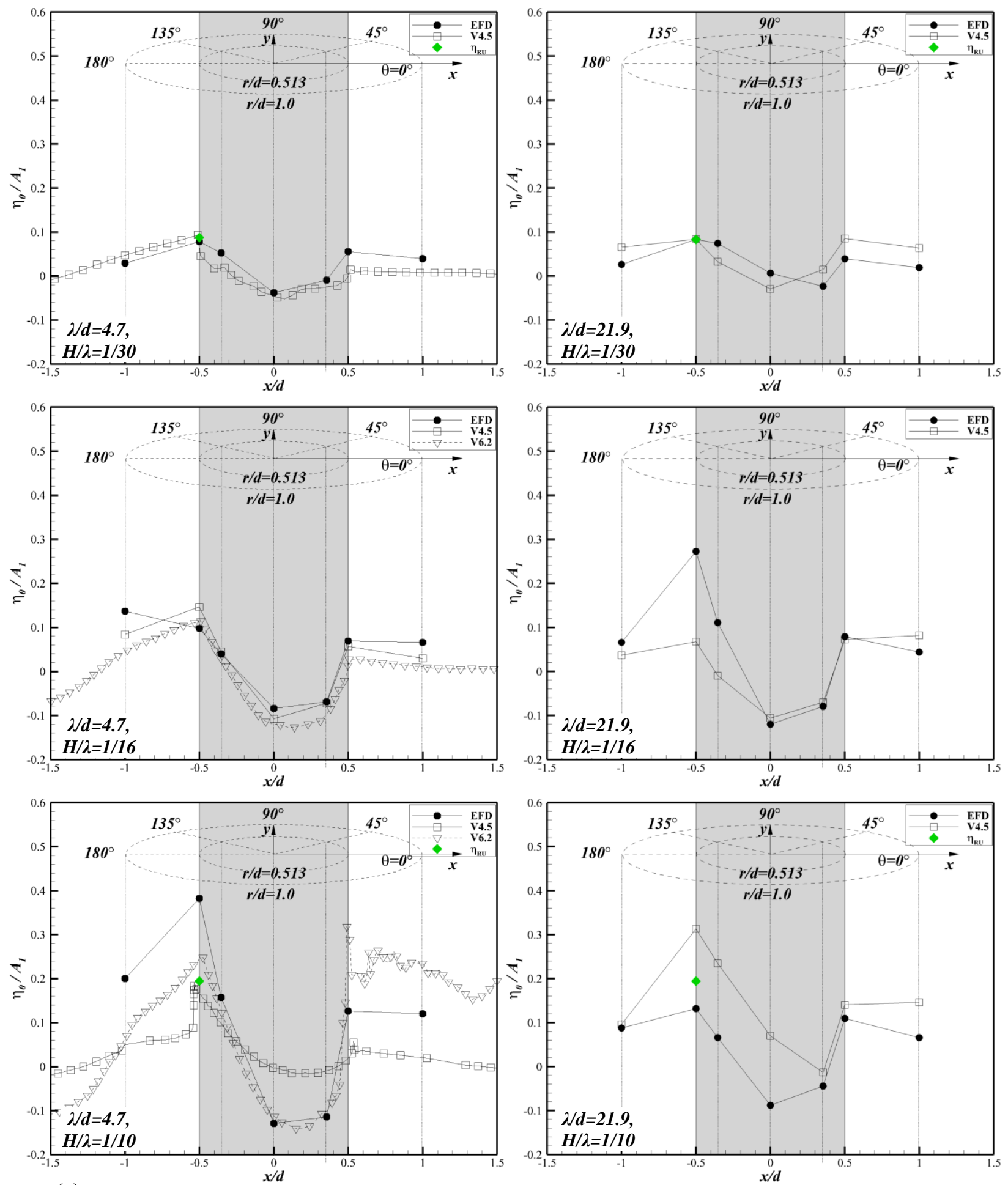

(a)

(b)

Figure 7 Mean wave profile for 1C: (a) short wave case, (b) long wave case; $\eta_{0}$ calculated along centerline $(y=0)$ of computational domain for off-body 


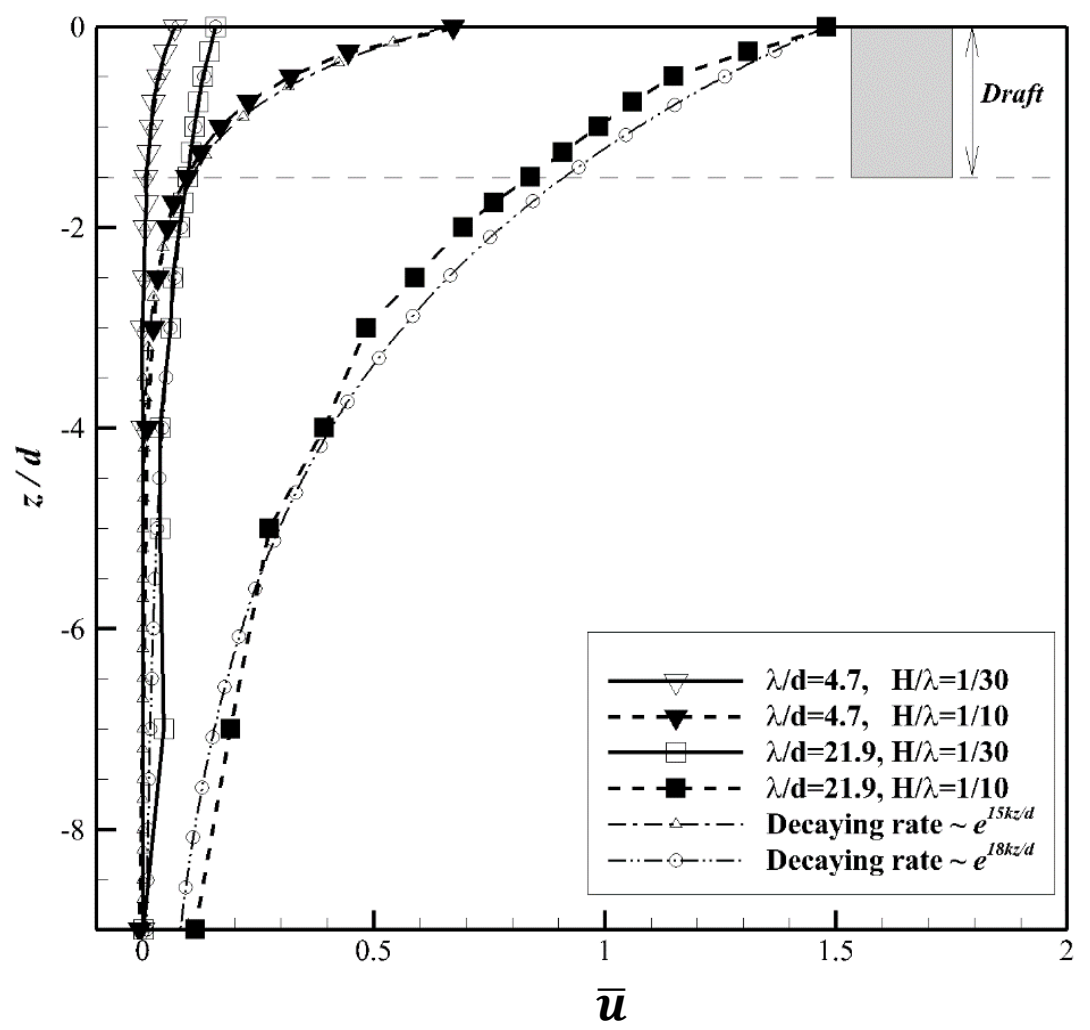

Figure 8 Mean horizontal velocity distribution according to vertical depth 

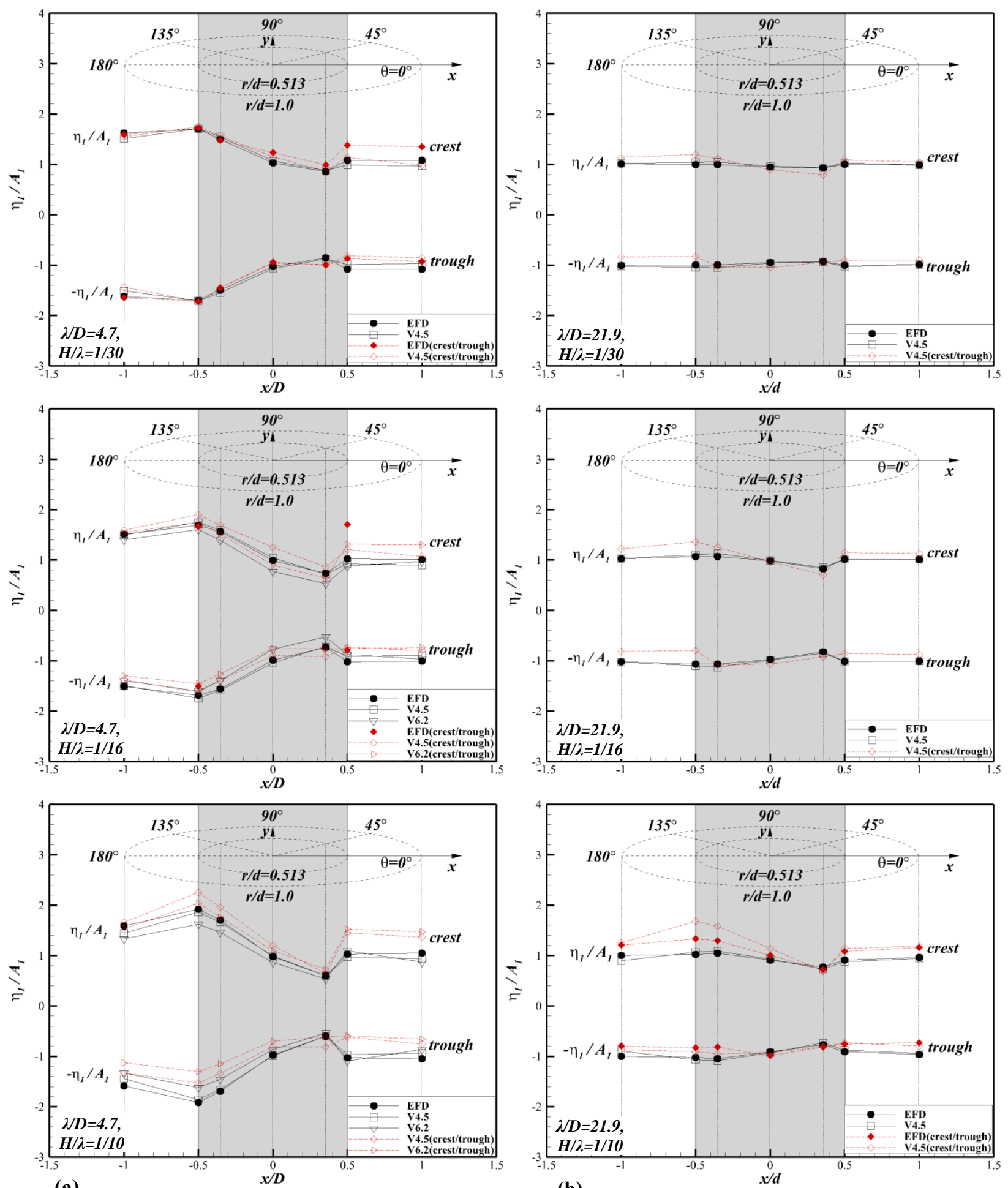

(a)

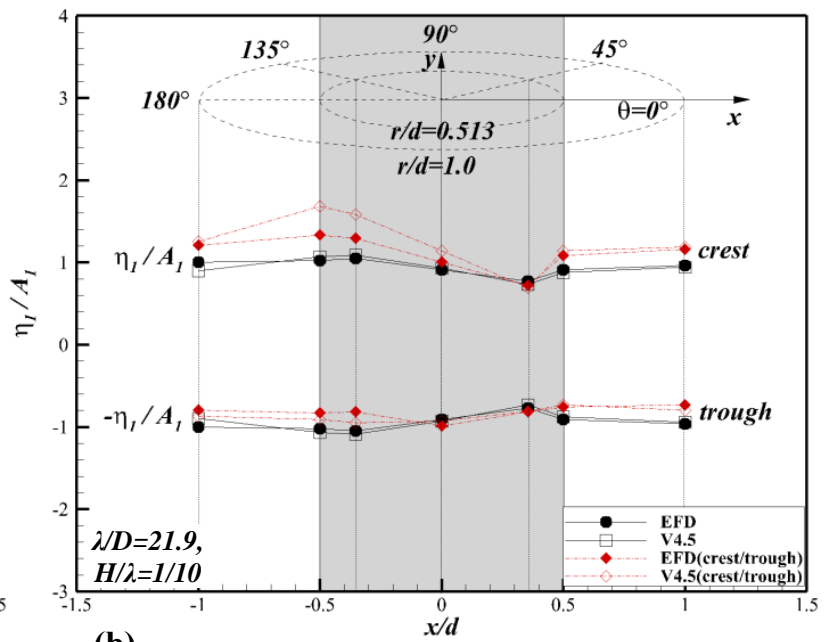

(b)

Figure $91^{\text {st }}$ harmonics of wave elevation for 1C: (a) short wave case, (b) long wave case 

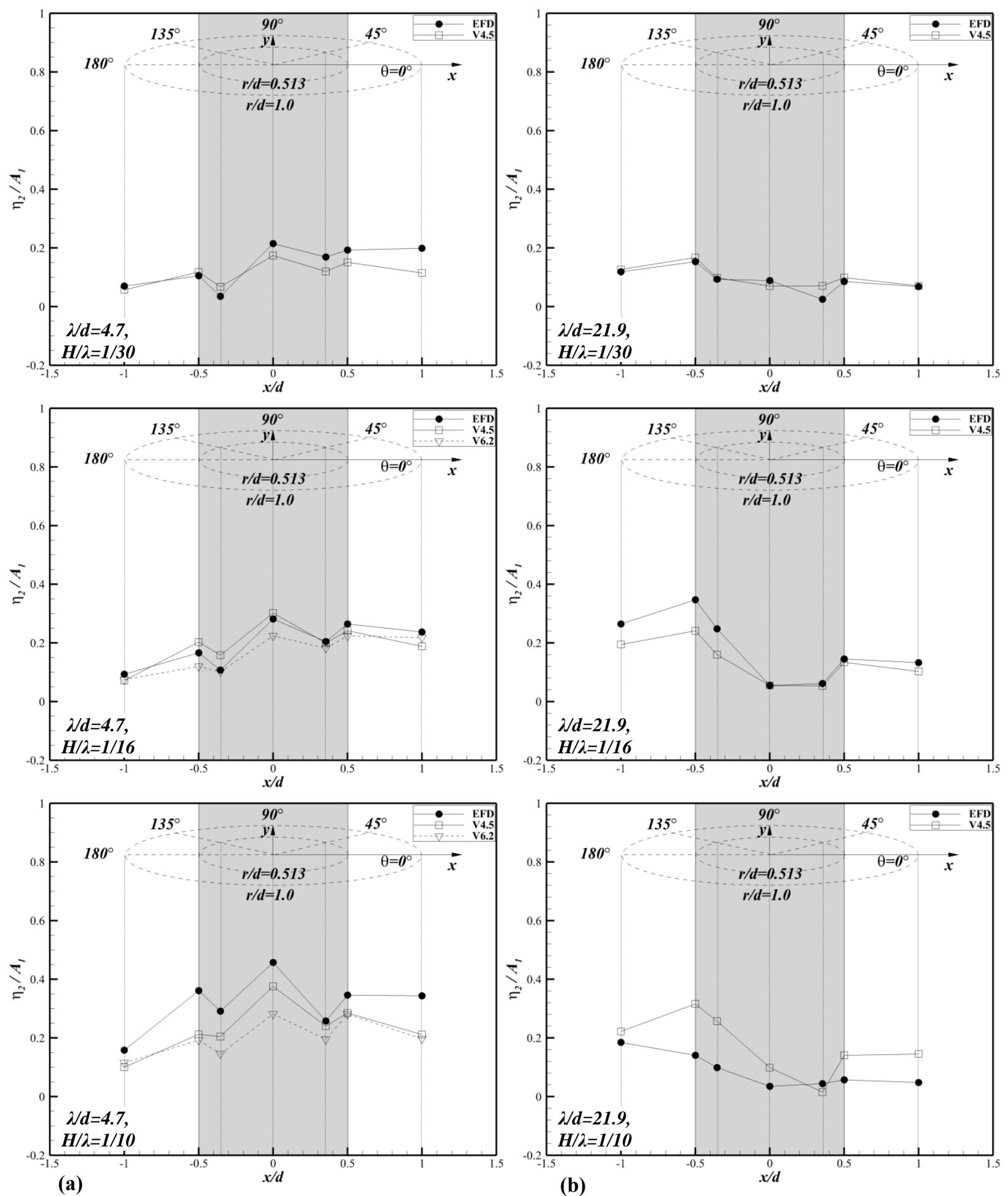

Figure $102^{\text {nd }}$ harmonics of wave elevation for 1C: (a) short wave case, (b) long wave case 


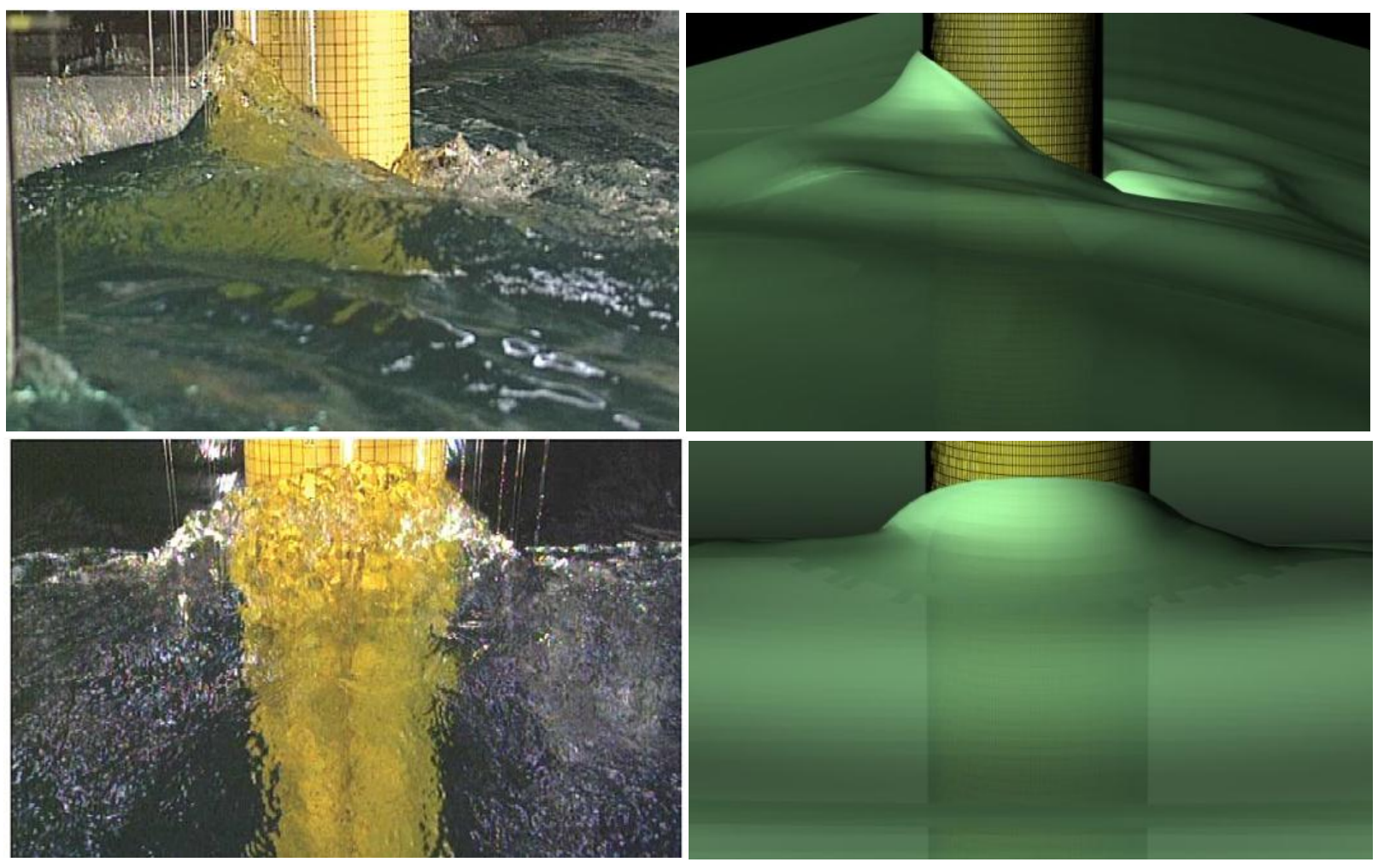

Figure 11 Comparison of instantaneous wave field between EFD and $\mathrm{CFD}_{\mathrm{v} 4.5}(1 \mathrm{C}, \lambda / d=21.9, H / \lambda=1 / 10)$ : photo from experiment [5]; (upper) side view, (lower) upwave view

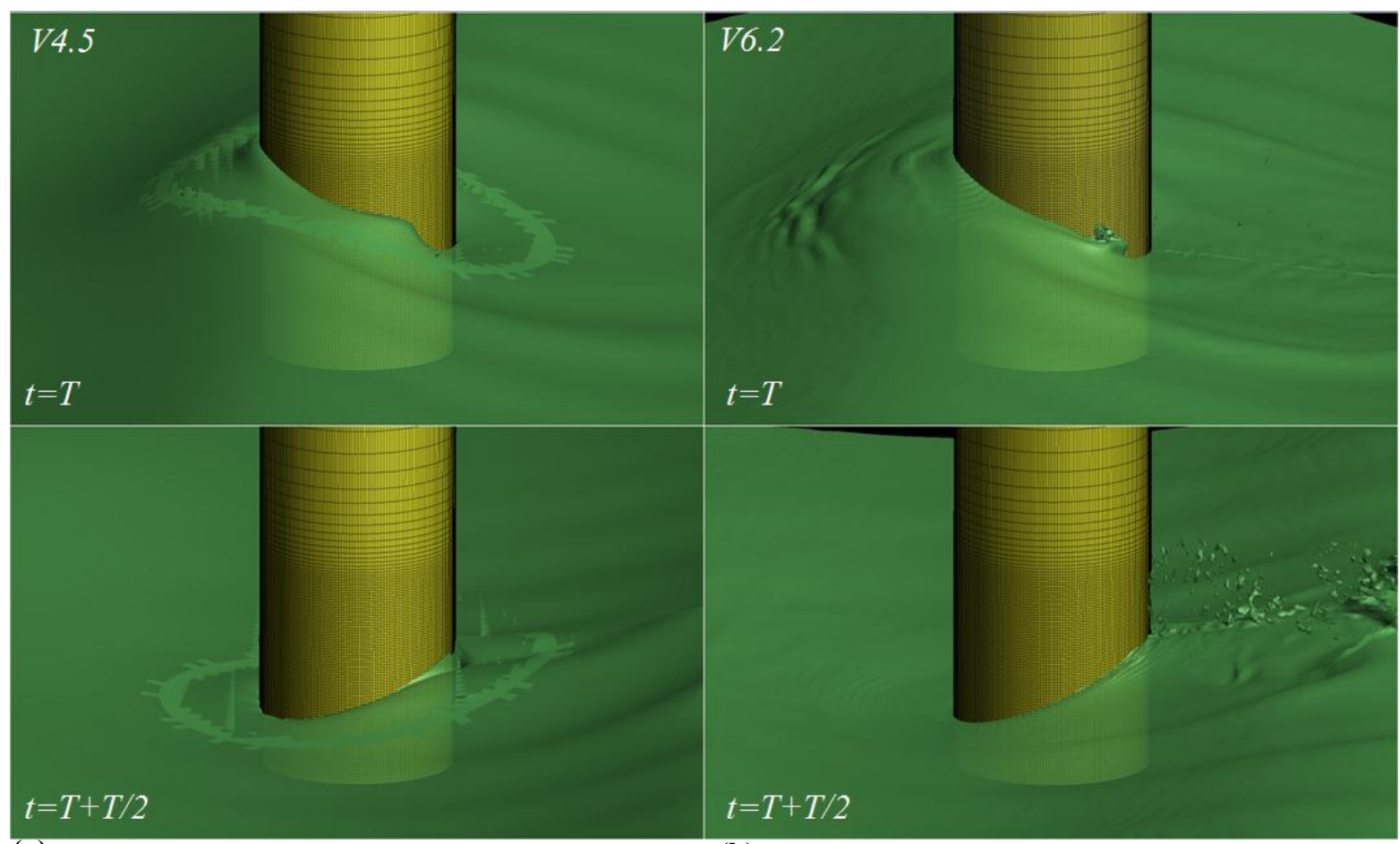

(a)

(b)

Figure 12 Comparison of instantaneous air/water interface profiles of $\mathrm{CFD}_{\mathrm{V} 4.5}$ and $\mathrm{CFD}_{\mathrm{v} 6.2}(1 \mathrm{C}, \lambda / d=4.7, H / \lambda=1 / 10)$ : (a) V4.5, (b) V6.2; $t=T$ and $t=T+T / 2$ when wave crest and trough meets cylinder, respectively 

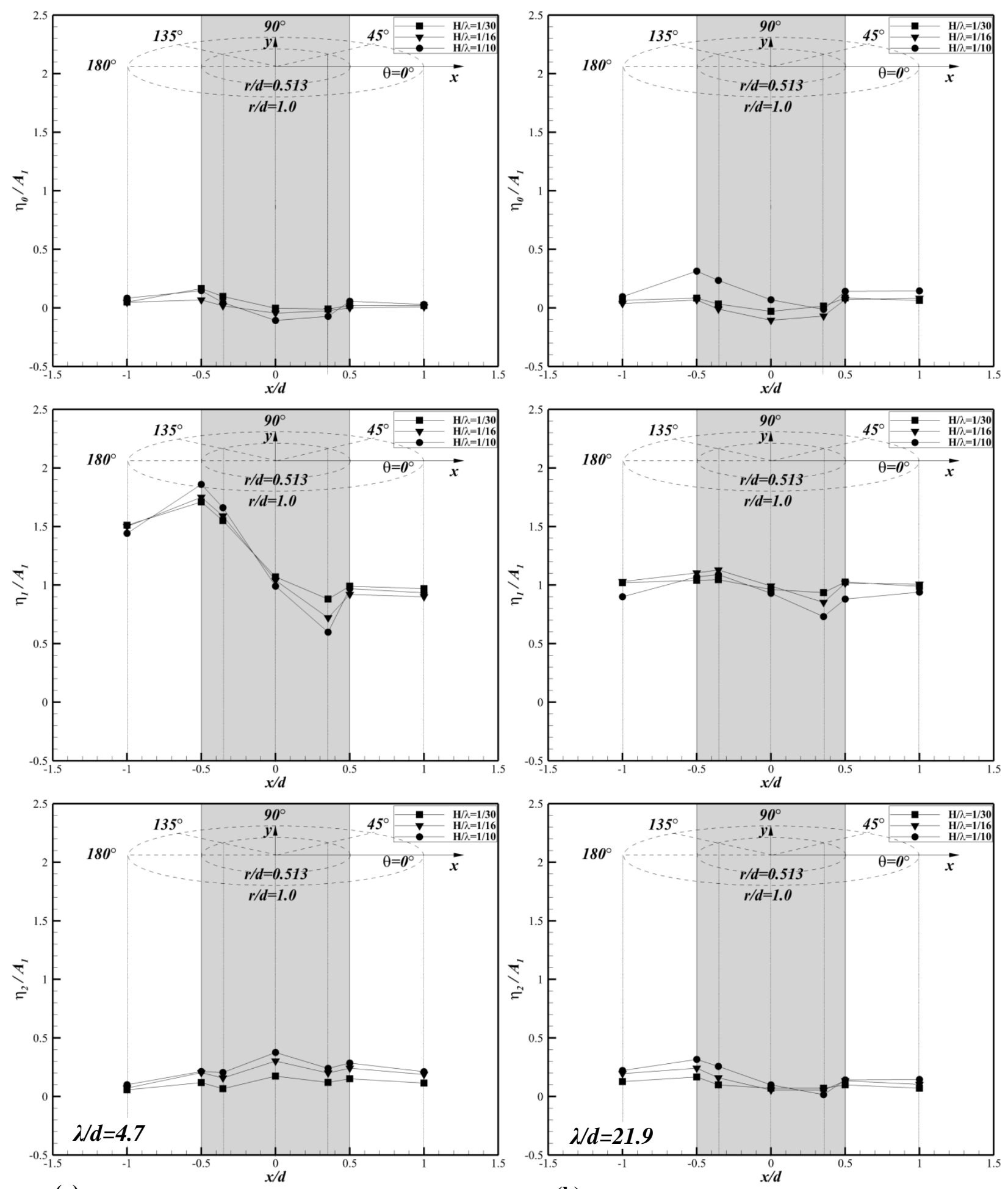

(a)

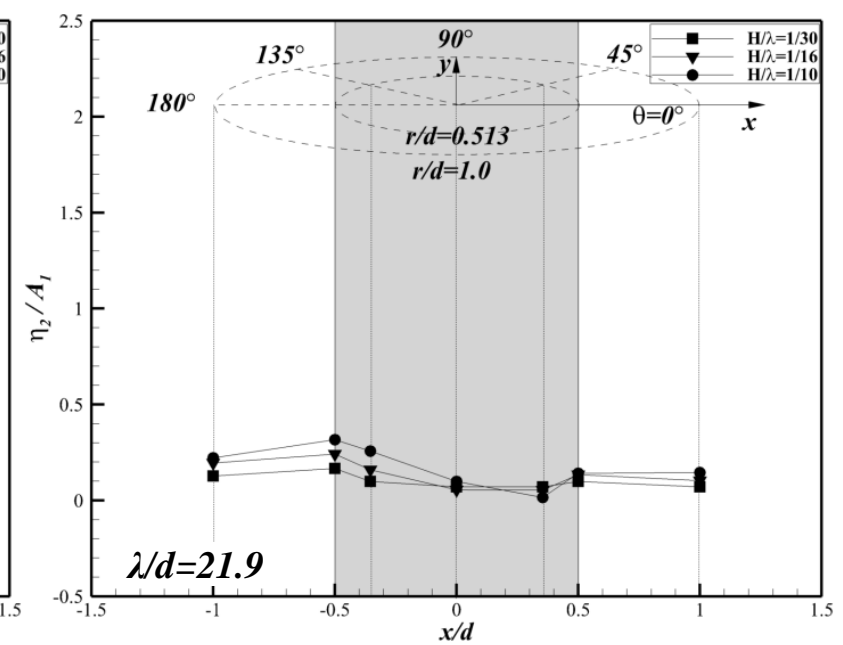

(b)

Figure 13 Mean, $1^{\text {st }}$ and $2^{\text {nd }}$ harmonics of wave elevation for different wave steepness (1C): (a) short wave cases, $\lambda / d=4.7$, (b) long wave cases, $\lambda / d=21.9$ 


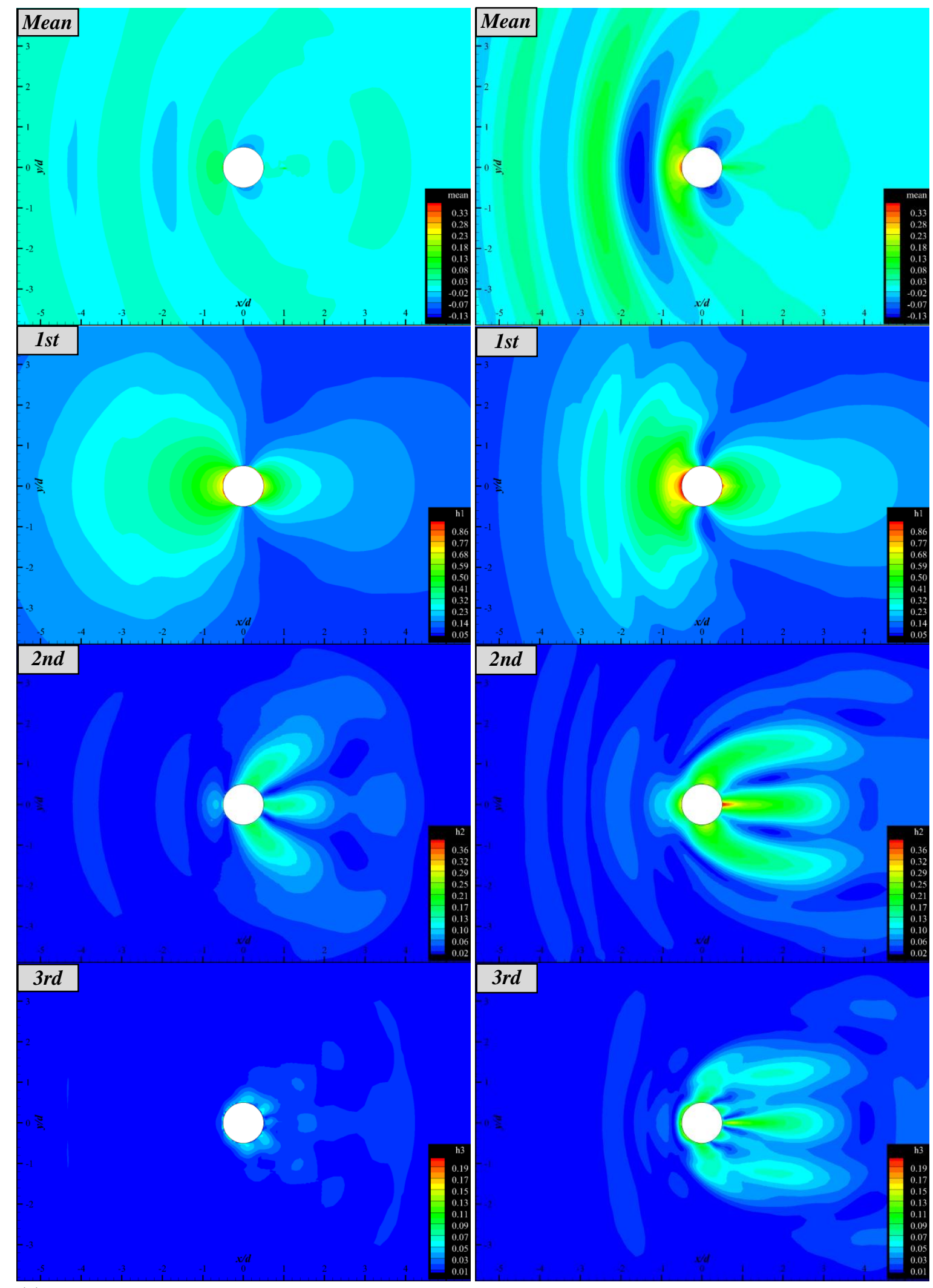

(a) (b)

Figure 14 Contours of harmonics for diffracted wave, $\eta_{D} / A_{1}=\left(\eta_{T}-\eta_{N C}\right) / A_{1}(1 \mathrm{C}, \lambda / d=4.7)$ : (a) $H / \lambda=1 / 30$, (b) $H / \lambda=1 / 10$; twenty contour levels determined to be suitable for the min. and max. values 


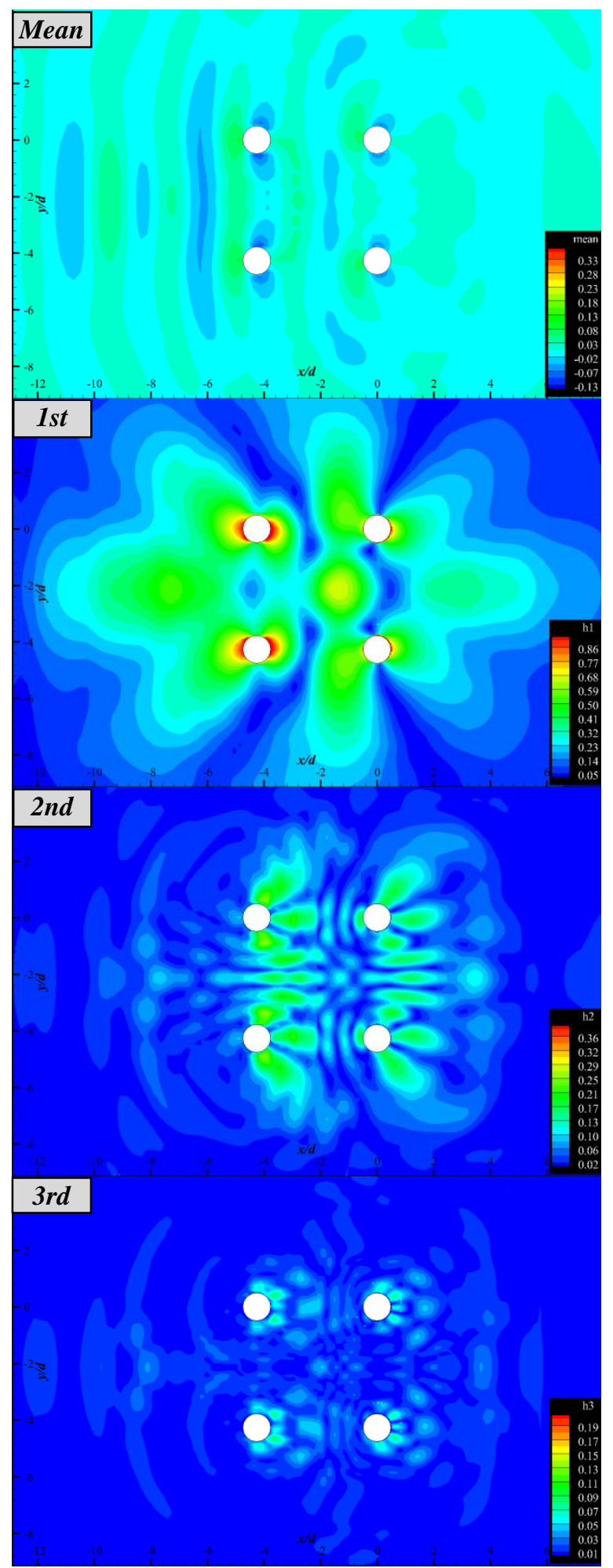

Figure 15 Contours of harmonics for diffracted wave, $\eta_{D} / A_{1}=\left(\eta_{T}-\eta_{N C}\right) / A_{1}(4 \mathrm{C}, \lambda / d=4.7, H / \lambda=1 / 30)$ ; twenty contour levels determined to be suitable for the min. and max. values 


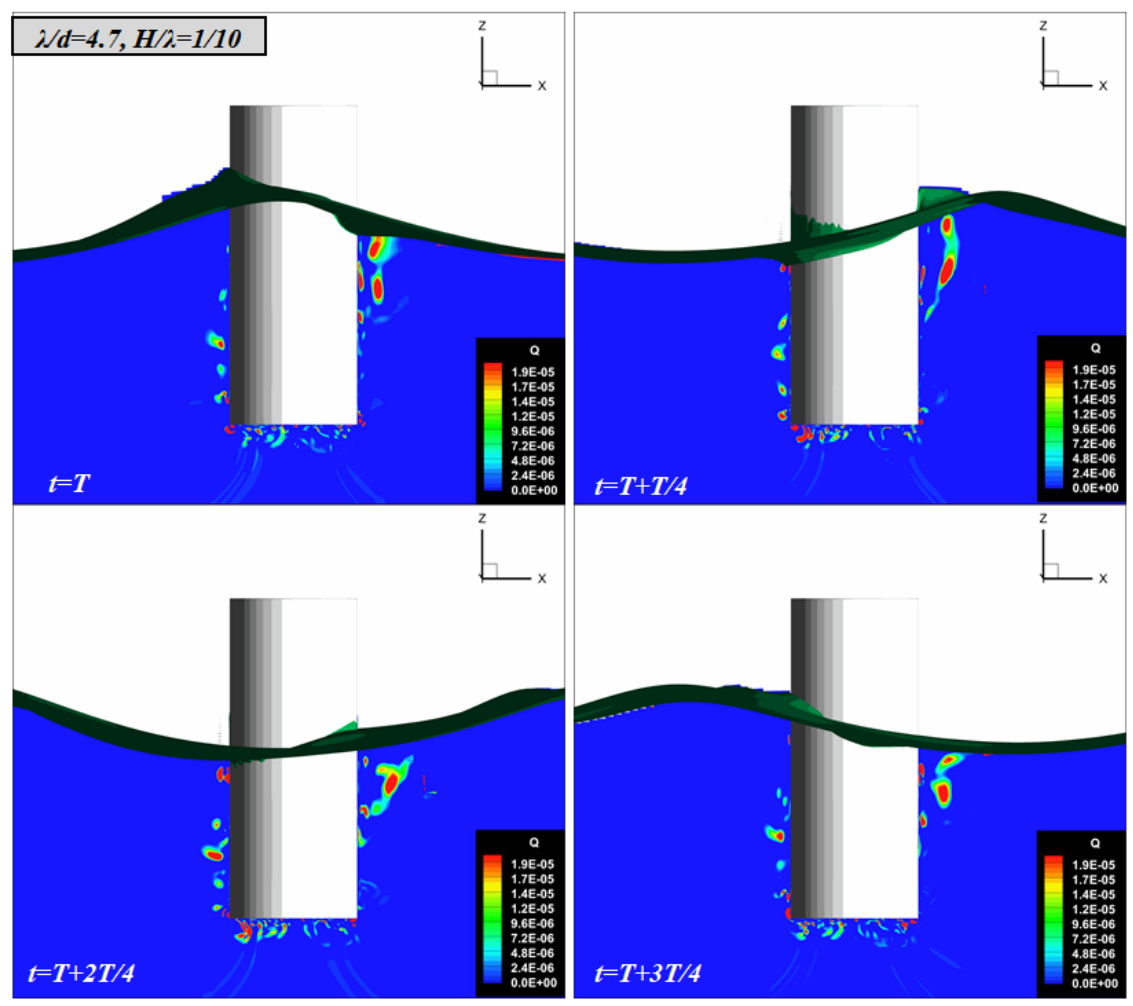

Figure 16 Instantaneous air/water interface and Q-criterion contour near the cylinder $(1 \mathrm{C}, \lambda / d=4.7, H / \lambda=1 / 10)$

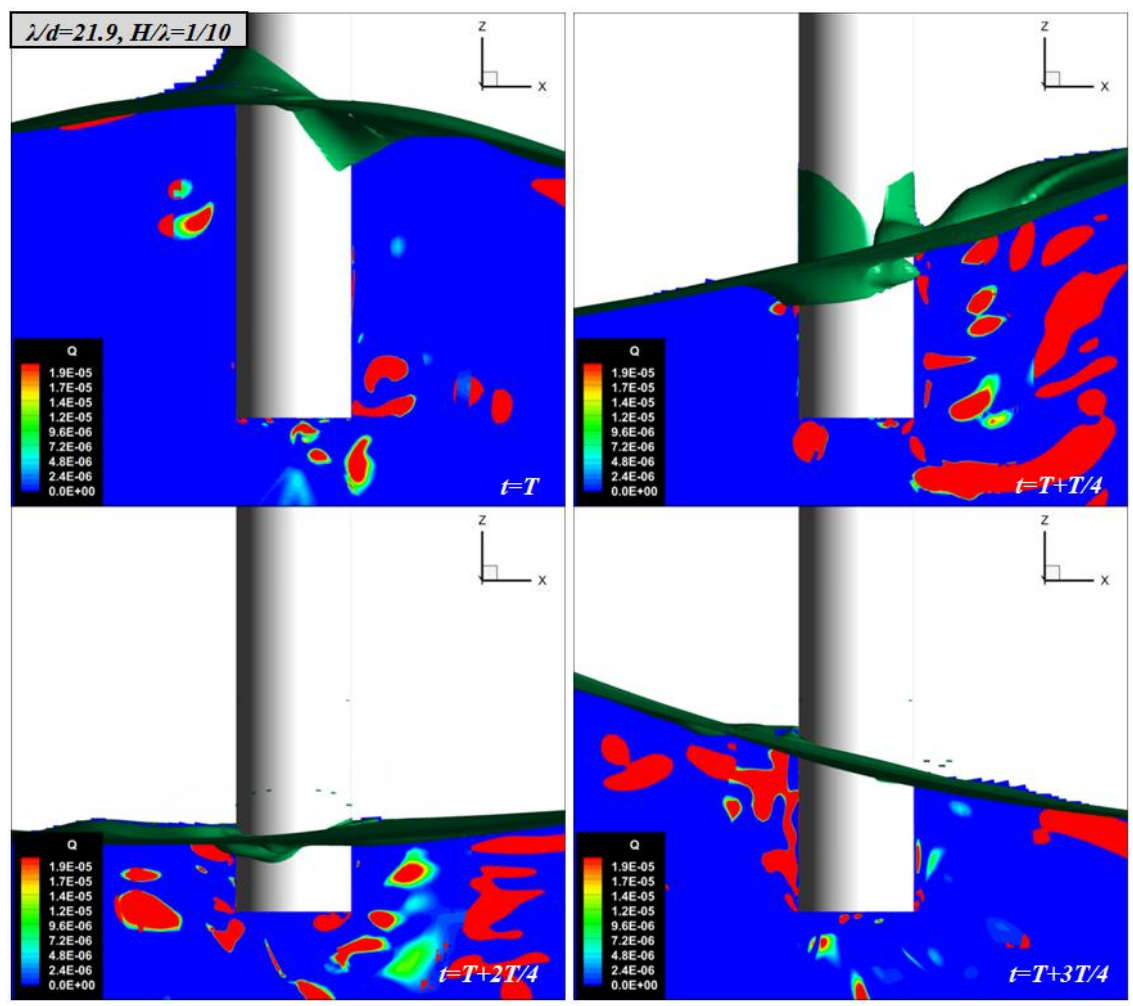

Figure 17 Instantaneous air/water interface and Q-criterion near the cylinder $(1 \mathrm{C}, \lambda / d=21.9, H / \lambda=1 / 10)$ 


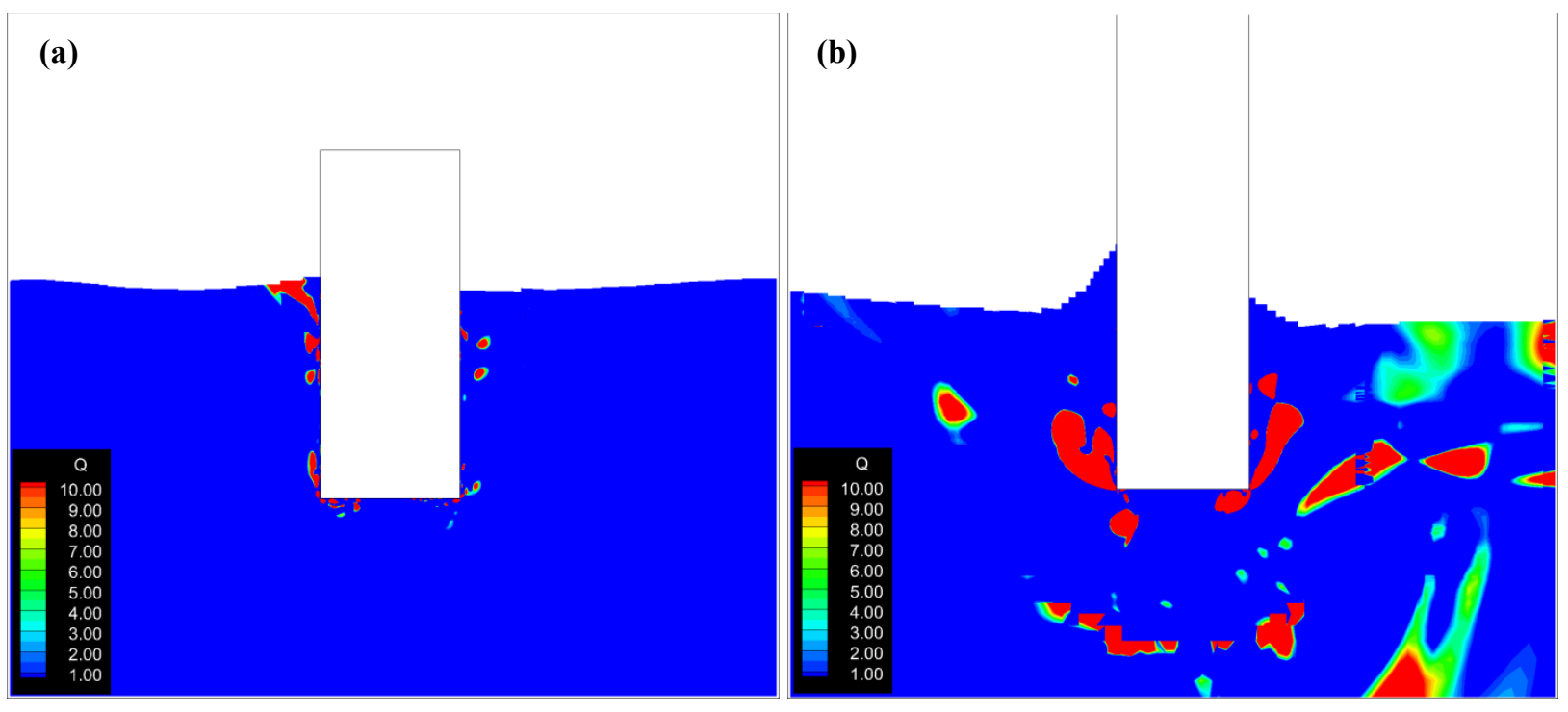

Figure 18 Mean air/water interface and Q-criterion near the cylinder: (a) $\lambda / d=4.7, H / \lambda=1 / 10$, (b) $\lambda / d=21.9, H / \lambda=1 / 10$ 
Table 1 Main particulars of single/four cylinders and facility biases uncertainty $\left(\mathrm{U}_{\mathrm{FB}}\right)$

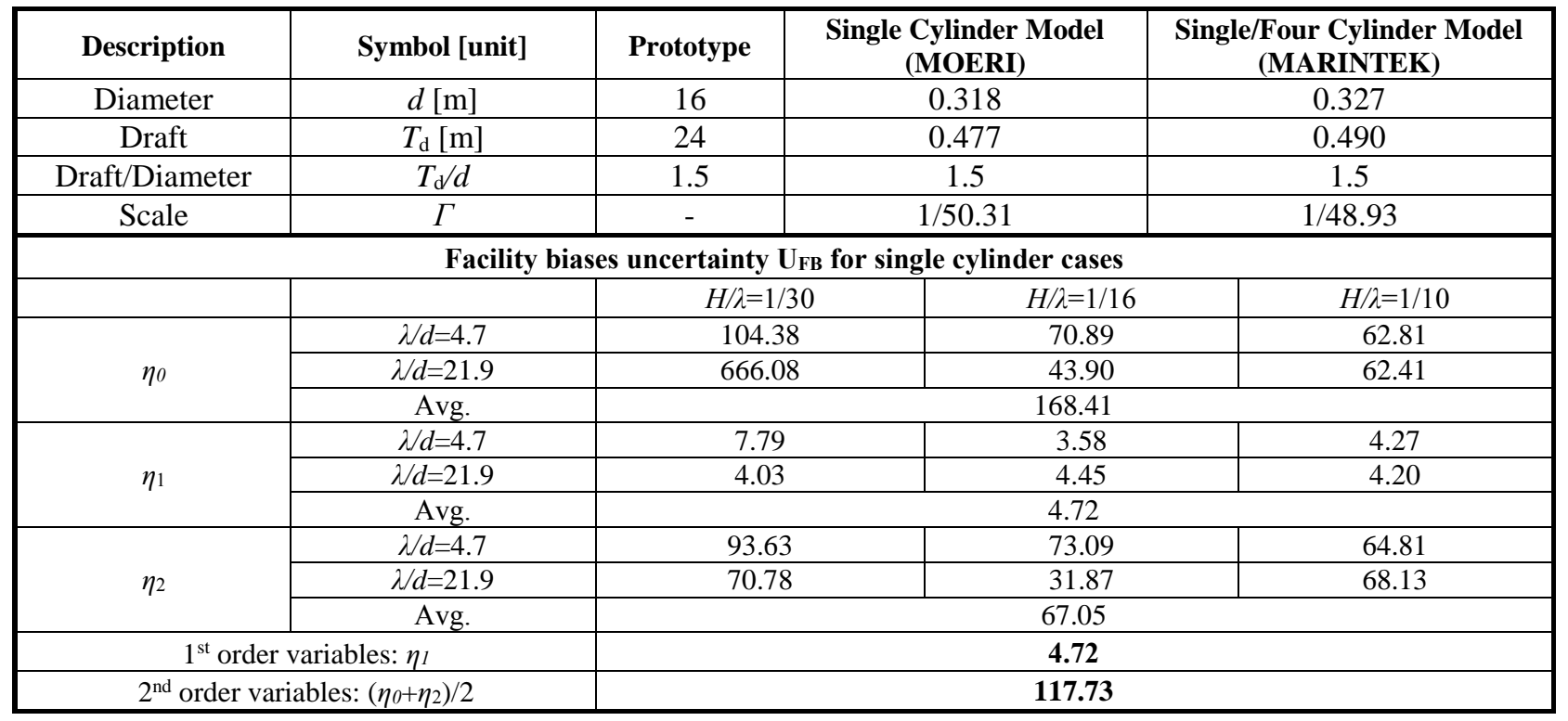

Table 2 Test matrix for CFD simulations

\begin{tabular}{|c|c|c|c|c|c|c|c|c|c|c|c|c|c|}
\hline \multirow{2}{*}{$\begin{array}{c}\text { Body/Code/ } \\
\text { Turbulence } \\
\text { model }\end{array}$} & \multicolumn{3}{|c|}{ Grid } & \multirow[b]{2}{*}{$\lambda / d$} & \multirow[b]{2}{*}{$T[\mathrm{~s}]$} & \multirow[b]{2}{*}{$f[\mathrm{~Hz}]$} & \multirow[b]{2}{*}{$H / \lambda$} & \multirow[b]{2}{*}{$R e$} & \multirow[b]{2}{*}{$\mathrm{Fr}$} & \multicolumn{4}{|c|}{ Wave resolution } \\
\hline & & Domain size & $\begin{array}{c}\text { Total grid } \\
\text { points }\end{array}$ & & & & & & & Grid type & $\lambda / \Delta x$ & $H / \Delta z$ & $T / \Delta t$ \\
\hline \multirow{16}{*}{$\begin{array}{c}\text { 1C / V4.5 / } \\
\text { RANS }\end{array}$} & \multirow{3}{*}{ G1 } & \multirow{3}{*}{$\begin{array}{c}\text { Large } \\
-3.0 \lambda<x<3.0 \lambda \\
-1.5 \lambda<y<1.5 \lambda \\
-2.0 \lambda<z<2.5 \mathrm{~d} \\
\end{array}$} & \multirow{3}{*}{$\begin{array}{c}24 \mathrm{M} \\
\text { Medium } \\
\text { resolution }\end{array}$} & \multirow{3}{*}{4.7} & \multirow{3}{*}{7} & \multirow{3}{*}{0.143} & $1 / 30$ & $7.34 \mathrm{E}+05$ & 0.0048 & Background & 76 & 30 & 548 \\
\hline & & & & & & & $1 / 16$ & $2.58 \mathrm{E}+06$ & 0.0168 & Background & 76 & 56 & 548 \\
\hline & & & & & & & $1 / 10$ & $6.60 \mathrm{E}+06$ & 0.0431 & Background & 76 & 90 & 548 \\
\hline & \multirow{9}{*}{ G2 } & \multirow{9}{*}{$\begin{array}{c}\text { Large } \\
-3.0 \lambda<x<3.0 \lambda \\
-1.5 \lambda<y<1.5 \lambda \\
-2.0 \lambda<z<6.0 \mathrm{~d}\end{array}$} & \multirow{9}{*}{$\begin{array}{c}23 \mathrm{M} \\
\text { Medium } \\
\text { resolution }\end{array}$} & \multirow{9}{*}{21.9} & \multirow{9}{*}{15} & \multirow{9}{*}{0.067} & \multirow{3}{*}{$1 / 30$} & \multirow{3}{*}{$1.57 \mathrm{E}+06$} & \multirow{3}{*}{0.0103} & Background & 76 & 20 & \multirow{3}{*}{1174} \\
\hline & & & & & & & & & & Refinement 2 & 154 & 20 & \\
\hline & & & & & & & & & & Refinement 1 & 336 & 22 & \\
\hline & & & & & & & \multirow{3}{*}{$1 / 16$} & \multirow{3}{*}{$5.53 \mathrm{E}+06$} & \multirow{3}{*}{0.0361} & Background & 76 & 58 & \multirow{3}{*}{1174} \\
\hline & & & & & & & & & & Refinement 2 & 154 & 58 & \\
\hline & & & & & & & & & & Refinement 1 & 336 & 64 & \\
\hline & & & & & & & & & & Background & 76 & 92 & \\
\hline & & & & & & & $1 / 10$ & $1.42 \mathrm{E}+07$ & 0.0924 & Refinement 2 & 154 & 94 & 1174 \\
\hline & & & & & & & & & & Refinement 1 & 336 & 104 & \\
\hline & G3 & $\begin{array}{c}\text { Medium } \\
-2.0 \lambda<x<3.0 \lambda\end{array}$ & $\begin{array}{l}25 \mathrm{M} \\
\text { Fine }\end{array}$ & & & & & & & Background & 82 & 58 & 548 \\
\hline & J & $\begin{array}{l}-1.5 \lambda<y<1.5 \lambda \\
-2.0 \lambda<z<2.5 \mathrm{~d}\end{array}$ & resolution & & & & & & & Refinement 1 & 119 & 132 & 340 \\
\hline & G4 & \begin{tabular}{|c|} 
Medium \\
$-2.0 \lambda<x<3.0 \lambda$ \\
$-1.5 \lambda<y<1.5 \lambda$ \\
$-2.0 \lambda<z<2.5 \mathrm{~d}$ \\
\end{tabular} & $\begin{array}{c}21 \mathrm{M} \\
\text { Medium } \\
\text { resolution }\end{array}$ & 4.7 & 7 & 0.143 & $1 / 30$ & $7.34 \mathrm{E}+05$ & 0.0048 & Background & 76 & 30 & 548 \\
\hline & G5 & $\begin{array}{c}\text { Small } \\
-\lambda<x<3.0 \lambda \\
-1.5 \lambda<y<1.5 \lambda \\
-2.0 \lambda<z<2.5 \mathrm{~d} \\
\end{array}$ & $\begin{array}{c}13 \mathrm{M} \\
\text { Medium } \\
\text { resolution }\end{array}$ & & & & & & & Background & 76 & 30 & 548 \\
\hline $\begin{array}{c}\text { 1C/ V4.5 / } \\
\text { RANS }\end{array}$ & & $\begin{array}{c}\text { Very small } \\
-0.6 \lambda<x<1.2 \lambda\end{array}$ & $26 \mathrm{M}$ & & & & $1 / 16$ & & & Background & 159 & 60 & 548 \\
\hline $\begin{array}{c}\mathrm{C} / \mathrm{V} 4.5 / \\
\mathrm{DES}\end{array}$ & G6 & $\begin{array}{l}-0.7 \lambda<y<0.7 \lambda \\
-1.4 \lambda<z<0.4 \mathrm{~d} \\
\end{array}$ & $\begin{array}{l}\text { Very fine } \\
\text { resolution }\end{array}$ & 4.7 & 7 & 0.143 & $1 / 16$ & 2.58 & & Background & 159 & 60 & 2192 \\
\hline $\begin{array}{c}\text { 1C / V6.2 / } \\
\text { No Turbulence }\end{array}$ & G7 & $\begin{array}{c}\text { Medium } \\
-2.0 \lambda<x<2.0 \lambda\end{array}$ & $\begin{array}{c}11 \mathrm{M} \\
\text { Coarse }\end{array}$ & 4.7 & 7 & 0.143 & $1 / 16$ & $2.58 \mathrm{E}+06$ & 0.0168 & Background & 128 & 22.8 & 210 \\
\hline model & 01 & $\begin{array}{l}-2.0 \lambda<y<2.0 \lambda \\
-3.0 \lambda<z<3.0 \mathrm{~d}\end{array}$ & resolution & 4.1 & 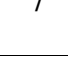 & 0.143 & $1 / 10$ & $6.60 \mathrm{E}+06$ & 0.0431 & Background & 128 & 36.4 & 520 \\
\hline $4 \mathrm{C} / \mathrm{V} 4.5 /$ & G8 & $\begin{array}{c}\text { Medium } \\
-2.0 \lambda<x<3.0 \lambda\end{array}$ & $\begin{array}{c}65 \mathrm{M} \\
\text { Medium }\end{array}$ & 47 & 7 & 0143 & $1 / 30$ & $734 \mathrm{~F}+05$ & 00048 & Background & 82 & 58 & 548 \\
\hline RANS & Uo & $\begin{array}{l}-1.5 \lambda<y<1.5 \lambda \\
-2.0 \lambda<z<2.5 \mathrm{~d}\end{array}$ & $\begin{array}{l}\text { Inedium } \\
\text { resolution }\end{array}$ & & & & & & & Refinement 1 & 119 & 132 & \\
\hline
\end{tabular}


Table 3 Effect of computational parameters on wave elevation and force

\begin{tabular}{|c|c|c|c|c|c|c|}
\hline & & & \multirow{2}{*}{$\begin{array}{c}\text { Grid sensitivity } \Delta \mathbf{G} \\
(\lambda / d=4.7, H / \lambda=1 / 30)\end{array}$} & \multicolumn{2}{|c|}{$\begin{array}{c}\text { Domain size } \Delta \mathbf{D} \\
(\lambda / d=4.7, H / \lambda=1 / 30)\end{array}$} & \multirow{2}{*}{$\begin{array}{c}\begin{array}{c}\text { Turbulence model } \Delta \mathbf{T M M} \\
(\lambda / d=4.7, H / \lambda=1 / 16)\end{array} \\
\Delta \mathrm{URANS} \% \mathrm{DES}\end{array}$} \\
\hline & & & & $\triangle \mathrm{D} 2 \% \mathrm{D} 1$ & $\triangle \mathrm{D} 3 \% \mathrm{D} 1$ & \\
\hline \multirow{2}{*}{\multicolumn{2}{|c|}{$\begin{array}{c}\text { Averaged wave height } \\
\text { (Avg. among } 10 \text { locations) }\end{array}$}} & $\overline{\eta_{c}}$ & 2.19 & 0.84 & 6.26 & 3.00 \\
\hline & & $\bar{\eta}_{t}$ & 4.13 & 1.66 & 5.15 & 1.35 \\
\hline \multirow{3}{*}{\multicolumn{2}{|c|}{$\begin{array}{l}\text { Harmonics of wave elevation } \\
\text { (Avg. among } 10 \text { locations) }\end{array}$}} & $\eta_{0}$ & 118.26 & 3.62 & 19.73 & 7.98 \\
\hline & & $\eta_{1}$ & 3.20 & 0.92 & 8.76 & 0.57 \\
\hline & & $\eta_{2}$ & 37.22 & 1.05 & 14.94 & 5.12 \\
\hline \multirow{3}{*}{\multicolumn{2}{|c|}{$\begin{array}{l}\text { Harmonics of } \\
\text { horizontal force }\end{array}$}} & $X_{0}$ & 3.80 & 4.65 & 29.07 & 6.38 \\
\hline & & $X_{I}$ & 0.18 & 0.97 & 7.39 & 0.33 \\
\hline & & $X_{2}$ & 20.00 & 6.12 & 24.49 & 12.00 \\
\hline \multicolumn{3}{|c|}{ wave height: $\left(\bar{\eta}_{c}+\bar{\eta}_{t}\right) / 2$} & 3.16 & 1.25 & 5.71 & 2.18 \\
\hline \multicolumn{3}{|c|}{$0^{\text {th }}$ harmonics: $\left(\eta_{0}+X_{0}\right) / 2$} & 61.03 & 4.14 & 24.4 & 7.18 \\
\hline \multicolumn{3}{|c|}{$1^{\text {st }}$ harmonics: $\left(\eta_{I}+X_{I}\right) / 2$} & 1.69 & 0.95 & 8.08 & 0.45 \\
\hline \multicolumn{3}{|c|}{$2^{\text {nd }}$ harmonics: $\left(\eta_{2}+X_{2}\right) / 2$} & 28.61 & 3.59 & 19.72 & 8.56 \\
\hline \multirow{2}{*}{$1^{\text {st }}$ order variables: } & \multicolumn{2}{|c|}{$\left(\bar{\eta}_{c}+\bar{\eta}_{t}+\eta_{I}\right) / 3$} & 3.17 & 1.14 & 6.72 & 1.64 \\
\hline & \multicolumn{2}{|c|}{$\left(\bar{\eta}_{c}+\bar{\eta}_{t}+\eta_{I}+X_{I}\right) / 4$} & 2.43 & 1.10 & 6.90 & 1.32 \\
\hline \multirow{2}{*}{$2^{\text {nd }}$ order variables: } & \multicolumn{2}{|c|}{$\left(\eta_{0}+\eta_{2}\right) / 2$} & 77.74 & 2.34 & 17.34 & 6.55 \\
\hline & \multicolumn{2}{|c|}{$\left(\eta_{0}+\eta_{2}+X_{0}+X_{2}\right) / 4$} & 44.82 & 3.87 & 22.06 & 7.87 \\
\hline
\end{tabular}

Table 4 Harmonics of horizontal and vertical forces for 1C

\begin{tabular}{|c|c|c|c|c|c|c|c|c|c|c|}
\hline \multicolumn{11}{|c|}{ Short wave case $(\lambda / d=4.7)$} \\
\hline & & \multicolumn{3}{|c|}{$H / \lambda=1 / 30$} & \multicolumn{3}{|c|}{$H / \lambda=1 / 16$} & \multicolumn{3}{|c|}{$H / \lambda=1 / 10$} \\
\hline & & EFD & CFD & E\%D & EFD & CFD & E\%D & EFD & CFD & E\%D \\
\hline \multirow{3}{*}{ Mean } & $X_{0} /\left(\rho g d^{2} A_{1} / 4\right)$ & 0.639 & 0.486 & 23.940 & 0.603 & 0.590 & 2.224 & 0.730 & 0.480 & 34.146 \\
\hline & $Z_{0} /\left(\rho g d^{2} A_{1} / 4\right)$ & -0.162 & 0.031 & 119.421 & -0.034 & -0.027 & 19.875 & -0.099 & -0.034 & 65.540 \\
\hline & average & & & 71.681 & & & 11.049 & & & 49.843 \\
\hline \multirow{6}{*}{$1^{s t}$} & $X_{1} /\left(\rho g d^{2} A_{1} / 4\right)$ & 5.583 & 4.881 & 12.586 & 5.069 & 3.727 & 26.489 & 5.146 & 4.773 & 7.241 \\
\hline & $Z_{1} /\left(\rho g d^{2} A_{1} / 4\right)$ & 0.401 & 0.256 & 36.105 & 0.384 & 0.304 & 20.626 & 0.407 & 0.303 & 25.513 \\
\hline & average & & & 24.345 & & & 23.558 & & & 16.377 \\
\hline & $X_{\varepsilon 1}\left[\right.$ deg.] ${ }^{\ddagger}$ & -29.37 & -42.50 & 3.65 & - & - & - & - & - & - \\
\hline & $Z_{\varepsilon 1}$ [deg.] & -6.08 & -7.90 & 0.51 & - & - & - & - & - & - \\
\hline & average & & & 2.08 & & & - & & & - \\
\hline \multirow{3}{*}{$2^{n d}$} & $X_{2} /\left(\rho g d^{2} A_{1} / 4\right)$ & 0.461 & 0.374 & 18.720 & 0.380 & 0.414 & -9.061 & 0.329 & 0.485 & -47.428 \\
\hline & $Z_{2} /\left(\rho g d^{2} A_{1} / 4\right)$ & 0.259 & 0.446 & -72.413 & 0.230 & 1.029 & -348.143 & 0.262 & 0.183 & 30.009 \\
\hline & average & & & 45.567 & & & 178.602 & & & 38.719 \\
\hline \multicolumn{11}{|c|}{ Long wave case $(\lambda d=21.9)$} \\
\hline & & \multicolumn{3}{|c|}{$H / \lambda=1 / 30$} & \multicolumn{3}{|c|}{$H / \lambda=1 / 16$} & \multicolumn{3}{|c|}{$H / \lambda=1 / 10$} \\
\hline & & EFD & CFD & E\%D & EFD & CFD & E\%D & EFD & CFD & E\%D \\
\hline \multirow{3}{*}{ Mean } & $X_{0} /\left(\rho g d^{2} A_{1} / 4\right)$ & 0.078 & 0.105 & -35.206 & 0.132 & 0.109 & 17.042 & 0.149 & 0.085 & 42.771 \\
\hline & $Z_{0} /\left(\rho g d^{2} A_{1} / 4\right)$ & -0.080 & -0.322 & -301.408 & -0.124 & -0.111 & 9.806 & -0.144 & -0.124 & 14.378 \\
\hline & average & & & 168.307 & & & 13.424 & & & 28.575 \\
\hline \multirow{6}{*}{$1^{s t}$} & $X_{1} /\left(\rho g d^{2} A_{1} / 4\right)$ & 2.276 & 3.288 & -44.482 & 2.229 & 2.079 & 6.732 & 2.198 & 1.828 & 16.848 \\
\hline & $Z_{1} /\left(\rho g d^{2} A_{1} / 4\right)$ & 1.965 & 2.827 & -43.912 & 2.044 & 1.801 & 11.898 & 2.188 & 1.701 & 22.263 \\
\hline & average & & & 44.197 & & & 9.315 & & & 19.555 \\
\hline & $X_{\varepsilon 1}$ [deg.] & - & - & - & - & - & - & -134.15 & -137.58 & 0.95 \\
\hline & $Z_{\varepsilon 1}$ [deg.] & - & - & - & - & - & - & -127.66 & -137.45 & 2.72 \\
\hline & average & & & - & & & - & & & 1.84 \\
\hline \multirow{3}{*}{$2^{n d}$} & $X_{2} /\left(\rho g d^{2} A_{1} / 4\right)$ & 0.737 & 1.326 & -80.000 & 0.702 & 0.410 & 41.504 & 0.598 & 0.291 & 51.295 \\
\hline & $Z_{2} /\left(\rho g d^{2} A_{1} / 4\right)$ & 0.027 & 0.105 & -284.259 & 0.032 & 0.046 & -46.137 & 0.038 & 0.031 & 19.306 \\
\hline & average & & & 182.130 & & & 43.821 & & & 35.300 \\
\hline
\end{tabular}

\footnotetext{
${ }^{\ddagger}$ Error percentage for phase is defined as $\mathrm{E} \% 360^{\circ}=(\mathrm{D}-\mathrm{S}) \% 360^{\circ}$
} 
Table 5 Harmonics of wave elevations for $1 \mathrm{C}$ and $4 \mathrm{C}$

\begin{tabular}{|c|c|c|c|c|c|c|c|c|c|}
\hline & & & & $\eta_{0} / A_{1}$ & $\eta_{1} / A_{1}$ & $\eta_{\varepsilon 1}$ [deg.] $^{\S}$ & $\eta_{2} / A_{1}$ & $\bar{\eta}_{c} / A_{1}$ & $\bar{\eta}_{t} / A_{1}$ \\
\hline \multirow{9}{*}{$\begin{array}{c}1 \mathrm{C}, \lambda / d=4.7 \\
H / \lambda=1 / 30\end{array}$} & \multirow{9}{*}{$E \% D_{\mathrm{V} 4.5}$} & \multirow{3}{*}{$\begin{array}{l}r / d=0.513 \\
\text { (on-body) }\end{array}$} & $\mathrm{Avg}_{\text {weather }}$ & 33.84 & 1.97 & 3.79 & 51.19 & 3.96 & 0.22 \\
\hline & & & $\mathrm{Avg}_{\text {leeward }}$ & 232.49 & 5.08 & 5.17 & 24.95 & 15.22 & 3.59 \\
\hline & & & Avg on-body $_{1}$ & 122.77 & 3.66 & 4.57 & 34.34 & 9.02 & 1.98 \\
\hline & & \multirow{3}{*}{$\begin{array}{c}r / d=1.0 \\
\text { (off-body) }\end{array}$} & Avg $_{\text {weather }}$ & 49.95 & 6.57 & 5.44 & 41.56 & 4.75 & 8.45 \\
\hline & & & Avg $g_{\text {leeward }}$ & 227.66 & 8.18 & 2.18 & 31.49 & 22.16 & 16.93 \\
\hline & & & $\mathrm{Avg}_{\text {off-body }}$ & 173.71 & 7.39 & 3.77 & 36.34 & 14.51 & 10.86 \\
\hline & & \multirow{3}{*}{ Total } & $\operatorname{Avg}_{\text {weather }}$ & 41.90 & 4.27 & 4.61 & 46.38 & 4.35 & 4.33 \\
\hline & & & $\mathrm{Avg}_{\text {leeward }}$ & 230.07 & 6.63 & 3.67 & 28.22 & 18.69 & 10.26 \\
\hline & & & $\mathrm{Avg}_{\text {total }}$ & 148.24 & 5.53 & 4.17 & 35.34 & 11.77 & 6.42 \\
\hline \multirow{9}{*}{$\begin{array}{c}1 \mathrm{C}, \lambda / d=21.9 \\
H / \lambda=1 / 10\end{array}$} & \multirow{9}{*}{ E\%Dv4.5 } & \multirow{3}{*}{$\begin{array}{l}r / d=0.513 \\
\text { (on-body) }\end{array}$} & $\operatorname{Avg}_{\text {weather }}$ & 10.75 & 4.06 & 0.91 & 142.50 & 24.31 & 12.33 \\
\hline & & & $\mathrm{Avg}_{\text {leeward }}$ & 31.90 & 4.30 & 2.61 & 105.58 & 4.68 & 2.28 \\
\hline & & & $\mathrm{Avg}_{\text {on-body }}$ & 21.06 & 3.83 & 1.69 & 135.48 & 14.46 & 7.03 \\
\hline & & \multirow{2}{*}{$\begin{array}{c}r / d=1.0 \\
\text { (off-body) }\end{array}$} & $\mathrm{Avg}_{\text {weather }}$ & 68.18 & 10.74 & 2.91 & 60.12 & 4.13 & 6.65 \\
\hline & & & $\mathrm{Avg}_{\text {leeward }}$ & 131.44 & 3.47 & 3.07 & 119.64 & 10.97 & 5.96 \\
\hline & & \multirow{4}{*}{ Total } & Avg $_{\text {weather }}$ & 39.47 & 7.40 & 1.91 & 101.31 & 14.22 & 9.49 \\
\hline & & & $\mathrm{Avg}_{\text {shoulder }}$ & 30.00 & 2.60 & 1.87 & 139.38 & 18.01 & 5.49 \\
\hline & & & $\mathrm{Avg}_{\text {leeward }}$ & 81.67 & 3.89 & 2.84 & 112.61 & 7.83 & 4.12 \\
\hline & & & $\mathrm{Avg}_{\text {total }}$ & 54.46 & 5.03 & 2.28 & 113.44 & 12.42 & 6.54 \\
\hline \multirow{10}{*}{$\begin{array}{c}1 \mathrm{C}, \lambda / d=4.7 \\
H / \lambda=1 / 16\end{array}$} & E\%Dv4.5 & $\begin{array}{l}r / d=0.513 \\
\text { (on-body) }\end{array}$ & Avg & 32.94 & 6.68 & - & 15.21 & 21.91 & 7.96 \\
\hline & $E \% D_{V 6.2}$ & $\begin{array}{l}r / d=0.513 \\
\text { (on-body) }\end{array}$ & Avg $g_{\text {on-body }}$ & 16.27 & 9.53 & - & 22.15 & 14.37 & 3.75 \\
\hline & \multirow{8}{*}{$\Delta \mathrm{V} 6.2 \% \mathrm{~V} 4.5$} & \multirow{3}{*}{$\begin{array}{l}r / d=0.513 \\
\text { (on-body) }\end{array}$} & $\mathrm{Avg}_{\text {weather }}$ & 11.91 & 10.44 & - & 37.72 & 9.14 & 9.72 \\
\hline & & & $\mathrm{Avg}_{\text {leeward }}$ & 24.23 & 15.35 & - & 8.19 & 17.02 & 10.04 \\
\hline & & & Avg $_{\text {on-body }}$ & 22.16 & 15.51 & - & 23.44 & 16.06 & 11.20 \\
\hline & & \multirow{3}{*}{$\begin{array}{c}r / d=1.0 \\
\text { (off-body) }\end{array}$} & $\mathrm{Avg}_{\text {weather }}$ & 18.79 & 6.33 & - & 14.51 & 4.55 & 6.51 \\
\hline & & & $\mathrm{Avg}_{\text {leeward }}$ & 75.86 & 8.77 & - & 9.91 & 14.80 & 5.86 \\
\hline & & & Avg $_{\text {off-body }}$ & 54.66 & 9.35 & - & 11.51 & 12.03 & 7.91 \\
\hline & & \multirow{2}{*}{ Total } & $\mathrm{Avg}_{\text {weather }}$ & 15.35 & 8.38 & - & 26.11 & 6.85 & 8.11 \\
\hline & & & Avg $g_{\text {total }}$ & 38.41 & 12.43 & - & 17.48 & 14.05 & 9.56 \\
\hline \multirow{10}{*}{$\begin{array}{c}4 \mathrm{C}, \lambda / d=4.7 \\
H / \lambda=1 / 30\end{array}$} & \multirow{8}{*}{$E \% D_{V 4.5}$} & $\theta=180^{\circ}$ & $\operatorname{Avg}_{\theta=180^{\circ}}$ & 78.66 & 4.87 & 5.72 & 37.91 & 10.66 & 14.31 \\
\hline & & $\theta=225^{\circ}$ & $\operatorname{Avg}_{\theta=225^{\circ}}$ & 500.59 & 8.05 & 1.18 & 24.01 & 7.26 & 8.54 \\
\hline & & $\theta=270^{\circ}$ & $\operatorname{Avg}_{\theta=270^{\circ}}$ & 54.58 & 9.35 & 3.37 & 14.67 & 3.22 & 13.14 \\
\hline & & \multirow{5}{*}{ Total } & $\operatorname{Avg}_{r / d=0.503}$ & 76.80 & 12.14 & 1.02 & 31.59 & 9.79 & 18.73 \\
\hline & & & $\operatorname{Avg}_{r / d=0.592}$ & 91.49 & 6.43 & 5.73 & 31.76 & 8.19 & 11.60 \\
\hline & & & $\operatorname{Avg}_{r / d=0.797}$ & 131.23 & 5.62 & 2.10 & 19.01 & 5.15 & 6.74 \\
\hline & & & $\operatorname{Avg}_{r / d=1.0}$ & 545.57 & 5.50 & 4.85 & 19.76 & 5.05 & 10.92 \\
\hline & & & $\mathrm{Avg}_{\text {total }}$ & 211.27 & 7.42 & 3.43 & 25.53 & 7.05 & 12.00 \\
\hline & & & & \multicolumn{5}{|c|}{$1^{\text {st }}$ order variables } & $\begin{array}{l}2^{\text {nd }} \text { order } \\
\text { variables }\end{array}$ \\
\hline & & & & $\left(\bar{\eta}_{c}+\bar{\eta}_{t}\right) / 2$ & $\eta_{1}$ & $\eta_{\varepsilon 1}$ [deg.] & $\left(\overline{\boldsymbol{\eta}}_{c}+\overline{\boldsymbol{\eta}}_{t}+\boldsymbol{\eta}_{1}\right) / \mathbf{3}$ & $\left(\overline{\boldsymbol{\eta}}_{c}+\overline{\boldsymbol{\eta}}_{t}+\boldsymbol{\eta}_{1}+\boldsymbol{\eta}_{\varepsilon I}\right) / 4$ & $\left(\eta_{0}+\eta_{2}\right) / 2$ \\
\hline $1 \mathrm{C}$ & E\%D 4.5 & Total & $\operatorname{Avg}_{1 \mathrm{C}}$ & 11.17 & 5.75 & 3.23 & 9.36 & 6.78 & 66.61 \\
\hline $1 \mathrm{C}$ & E\%DV6.2 & Total & $\operatorname{Avg}_{1 \mathrm{C}}$ & 9.06 & 9.53 & - & 9.22 & - & 19.21 \\
\hline $4 \mathrm{C}$ & E\%D $\mathrm{D}_{\mathrm{V} .5}$ & Total & $\mathrm{Avg}_{4 \mathrm{C}}$ & 9.53 & 7.42 & 3.43 & 8.82 & 7.47 & 118.40 \\
\hline $1 \mathrm{C}$ & OEC E\%D $\overline{\mathbf{E}}^{\text {*k }}$ & Total & $\operatorname{Avg}_{1 \mathrm{C}}$ & - & 5.63 & - & - & - & 141.03 \\
\hline
\end{tabular}

${ }^{\S}$ Error percentage for phase is defined as $\mathrm{E} \% 360^{\circ}=(\mathrm{D}-\mathrm{S}) \% 360^{\circ}$

${ }^{* *} \overline{\mathrm{E}}=\Delta \mathrm{CFD}_{\text {average }} \% \mathrm{EFD}_{\mathrm{MOERI}}$ 
Table 6 Comparison of wave elevations for $1 \mathrm{C}$ and $4 \mathrm{C}$ ( Cyl.A, downwave; $\lambda / d=4.7, H / \lambda=1 / 30$ )

\begin{tabular}{|c|c|c|c|c|}
\hline & \multicolumn{2}{|c|}{$\bar{\eta}_{c} / A_{1}$} & \multicolumn{2}{|c|}{$\bar{\eta}_{t} / A_{1}$} \\
\hline & $\Delta \mathrm{EFD}_{4 \mathrm{C}} \% \mathrm{EFD}_{1 \mathrm{C}}$ & $\triangle \mathrm{CFD}_{4 \mathrm{C}} \% \mathrm{CFD}_{1 \mathrm{C}}$ & $\Delta \mathrm{EFD}_{4 \mathrm{C}} \% \mathrm{EFD}_{1 \mathrm{C}}$ & $\triangle \mathrm{CFD}_{4 \mathrm{C}} \% \mathrm{CFD}_{1 \mathrm{C}}$ \\
\hline $\operatorname{Avg}_{\theta=180^{\circ}}$ & 8.82 & 5.25 & 16.93 & 4.32 \\
\hline $\operatorname{Avg}_{\theta=225^{\circ}}$ & 13.39 & 12.69 & 7.21 & 13.34 \\
\hline $\operatorname{Avg}_{\theta=270^{\circ}}$ & 25.26 & 17.34 & 5.81 & 10.46 \\
\hline $\operatorname{Avg}_{r=0.503 d}$ & 15.60 & 14.45 & 11.70 & 7.68 \\
\hline $\operatorname{Avg}_{r=1.0 d}$ & 16.05 & 9.07 & 8.27 & 11.06 \\
\hline Avg $_{\text {total }}$ & 15.82 & 11.76 & 9.98 & 9.37 \\
\hline
\end{tabular}

Table 7 Summary of parameters for separated vortices

\begin{tabular}{|c|c|c|c|c|c|c|c|c|c|c|c|c|}
\hline$T[\mathbf{s}]$ & $\lambda d$ & $H / \lambda$ & $A_{1} / T_{d}$ & $\lambda T_{d}$ & $u_{\zeta}[\mathrm{m} / \mathrm{s}]$ & $u_{T_{d}}[\mathrm{~m} / \mathrm{s}]$ & $\boldsymbol{u}_{\mathrm{T}_{\mathrm{d}}} / \boldsymbol{u}_{\zeta}[\%]$ & $T_{k}$ & $(T / 2) / T_{k}$ & $\boldsymbol{K}_{c}$ & $C_{d}$ & $C_{m}$ \\
\hline \multirow{3}{*}{7} & \multirow{3}{*}{4.7} & $1 / 30$ & 0.055 & & 1.14 & 0.16 & \multirow{3}{*}{13.9} & 58.5 & 0.06 & 0.50 & 0.33 & 1.33 \\
\hline & & $1 / 16$ & 0.100 & 3.19 & 2.15 & 0.30 & & 31.0 & 0.11 & 0.94 & - & - \\
\hline & & $1 / 10$ & 0.160 & & 3.43 & 0.48 & & 19.4 & 0.18 & 1.50 & - & - \\
\hline \multirow{3}{*}{15} & \multirow{3}{*}{21.9} & $1 / 30$ & 0.245 & & 2.45 & 1.60 & \multirow{3}{*}{65.1} & 27.2 & 0.28 & 2.30 & - & - \\
\hline & & $1 / 16$ & 0.460 & 14.65 & 4.60 & 3.00 & & 14.5 & 0.52 & 4.31 & - & - \\
\hline & & $1 / 10$ & 0.730 & & 7.36 & 4.79 & & 9.1 & 0.83 & 6.90 & 0.42 & 1.33 \\
\hline
\end{tabular}

